\title{
Algebraicity of cycles on smooth manifolds
}

\author{
Wojciech Kucharz
}

Published online: 19 August 2011

(C) The Author(s) 2011. This article is published with open access at Springerlink.com

\begin{abstract}
According to the Nash-Tognoli theorem, each compact smooth manifold $M$ is diffeomorphic to a nonsingular real algebraic set, called an algebraic model of $M$. It is interesting to investigate to what extent algebraic and differential topology of compact smooth manifolds can be transferred into the algebraic-geometric setting. Many results, examples and counterexamples depend on the detailed study of the homology classes represented by algebraic subsets of $X$, as $X$ runs through the class of all algebraic models of $M$. The present paper contains several new results concerning such algebraic homology classes. In particular, a complete solution in codimension 2 and strong results in codimensions 3 and 4.
\end{abstract}

Keywords Real algebraic set - Algebraic homology class - Algebraic model of a smooth manifold

Mathematics Subject Classification (2010) $\quad$ 14P05 $\cdot 14 \mathrm{P} 25 \cdot 57 \mathrm{R} 19$

\section{Introduction and main results}

There is a large research program whose goal is to transfer, as far as possible, algebraic and differential topology of compact smooth (of class $\mathcal{C}^{\infty}$ ) manifolds into the algebraic-geometric setting. The origins of this program go back to 1952 and the celebrated paper of J. Nash on real algebraic manifolds [53] (cf. also [16, Theorem 14.1.8]). Nash's result and conjectures inspired several mathematicians, but despite their efforts, no significant progress was made for 20 years (cf. [34] for historical

W. Kucharz $(\varangle)$

Institute of Mathematics, Jagiellonian University, ul. Profesora Łojasiewicza 6, 30-348 Kraków, Poland e-mail: Wojciech.Kucharz@im.uj.edu.pl 
remarks). A breakthrough came in 1973 due to Tognoli [63], who proved one of Nash's conjectures (cf. also [16, Theorem 14.1.10]). According to Tognoli's theorem, every compact smooth manifold $M$ is diffeomorphic to a nonsingular real algebraic set (in $\mathbb{R}^{n}$ for some $n$ ), called an algebraic model of $M$. A projective version of this theorem was proved in 1976 by King [35]. Actually, both [63] and [35] contain much stronger results, concerning approximation of smooth manifolds by algebraic sets, as suggested in [53]. Remarkable refinements of [35,63] can be found in the contributions from the 1980s and 1990s of two pairs of researchers, Akbulut-King [1-3,6-8] and Benedetti-Tognoli $[13,14]$. If some topological objects such as smooth submanifolds, vector bundles, homology or cohomology classes are attached to $M$, it is interesting to investigate whether or not there exists an algebraic model of $M$ on which the corresponding objects admit an algebraic description. Important positive results are known for smooth submanifolds [2,13] and vector bundles [13,14]. Contrary to initial expectations, expressed explicitly in $[2,3]$, the situation is drastically different for homology and cohomology classes, where obstructions appear [12,20,41,42,61]. This on the one hand imposes limitations and on the other hand leads to challenging problems considered below.

Let $X$ be a compact nonsingular real algebraic set. A homology class in $H_{d}(X ; \mathbb{Z} / 2)$ is said to be algebraic if it can be represented by a $d$-dimensional algebraic subset of $X$ (cf. [27] and [8,16,22]). The set $H_{d}^{\mathrm{alg}}(X ; \mathbb{Z} / 2)$ of all algebraic homology classes in $H_{d}(X ; \mathbb{Z} / 2)$ forms a subgroup. Early papers dealing with algebraic homology classes provided examples of $X$ with $H_{d}^{\text {alg }}(X ; \mathbb{Z} / 2) \neq H_{d}(X ; \mathbb{Z} / 2)$ for some $d, 1 \leq d \leq \operatorname{dim} X-1$ (cf. [3,14,15,36,54,57]). For technical reasons, it is often preferable to work with cohomology rather than homology. The subgroup $H_{\text {alg }}^{k}(X ; \mathbb{Z} / 2)$ of algebraic cohomology classes in $H^{k}(X ; \mathbb{Z} / 2)$ is by definition the inverse image of $H_{n-k}^{\text {alg }}(X ; \mathbb{Z} / 2)$ under the Poincare duality isomorphism $H^{k}(X ; \mathbb{Z} / 2) \rightarrow H_{n-k}(X ; \mathbb{Z} / 2)$, where $n=\operatorname{dim} X$. In particular, $H_{\text {alg }}^{n}(X ; \mathbb{Z} / 2)=$ $H^{n}(X ; \mathbb{Z} / 2)$. The direct sum

$$
H_{\text {alg }}^{*}(X ; \mathbb{Z} / 2)=\bigoplus_{k \geq 0} H_{\text {alg }}^{k}(X ; \mathbb{Z} / 2)
$$

is a subring of the cohomology ring $H^{*}(X ; \mathbb{Z} / 2)$, containing the Stiefel-Whitney classes $w_{k}(X)$ of $X$ for $k \geq 0$ (cf. [27] and, for purely topological proofs, [4,15, 56]). Consequently, $H_{\text {alg }}^{*}(X ; \mathbb{Z} / 2)$ contains the subring of $H^{*}(X ; \mathbb{Z} / 2)$ generated by $H^{n}(X ; \mathbb{Z} / 2)$ and $w_{k}(X)$ for $k \geq 0$. What other, if any, cohomology classes belong to $H_{\text {alg }}^{*}(X ; \mathbb{Z} / 2)$ depends in a very subtle way on the algebraic-geometric properties of $X$ (cf. $[21,31,49-51,58,65,66])$. The groups $H_{d}^{\text {alg }}(-; \mathbb{Z} / 2)$ and $H_{\text {alg }}^{k}(-; \mathbb{Z} / 2)$ are closely related via the cycle maps to the Chow groups of quasiprojective schemes over $\mathbb{R}$ and to the equivariant cohomology of the set of complex points of such schemes (cf. $[27,28,33,36,48,66])$. They play a crucial role in the research program described at the beginning (cf. [1,3-5, 8-18,20,23-26,37-48,55,56, 61,64] and, for a short survey, [22]).

Numerous results, examples and counterexamples in the papers just cited required information on algebraic homology and cohomology classes on various algebraic 
models of a given compact smooth manifold $M$. According to [19], $M$ has an uncountable family of pairwise nonisomorphic algebraic models whenever $\operatorname{dim} M \geq 1$. However, $M$ may not admit any algebraic model $X$ with $H_{\text {alg }}^{*}(X ; \mathbb{Z} / 2)=H^{*}(X ; \mathbb{Z} / 2)$ (see the remarks preceding Corollary 1.3). In order to avoid awkward repetitions, if $X$ is an algebraic model of $M$ and $\varphi: X \rightarrow M$ is a smooth diffeomorphism, the pair $(X, \varphi)$ will also be called an algebraic model of $M$. A subring $A$ of $H^{*}(M ; \mathbb{Z} / 2)$ (only subrings containing the identity element $1 \in H^{0}(M ; \mathbb{Z} / 2)$ are considered) is said to be algebraically realizable if there exists an algebraic model $(X, \varphi)$ of $M$ with $\varphi^{*}(A) \subseteq H_{\text {alg }}^{*}(X ; \mathbb{Z} / 2)$. An important algebraically realizable subring of $H^{*}(M ; \mathbb{Z} / 2)$ is identified in [13, Theorem 4, Remark 8]. It is the subring $A(M)$ generated by the Stiefel-Whitney classes of all real vector bundles on $M$ and the cohomology classes corresponding via the Poincaré duality to the homology classes represented by all compact smooth submanifolds of $M$. A conjecture posed in [12] asserts that every algebraically realizable subring of $H^{*}(M ; \mathbb{Z} / 2)$ is contained in $A(M)$. The conjecture is true if $\operatorname{dim} M \leq 7$, but in higher dimensions, it is not even known whether or not there is a largest algebraically realizable subring of $H^{*}(M ; \mathbb{Z} / 2)$ (cf. [41] for comments and conjectures).

The finer problem that of finding a characterization of the subrings $A$ of $H^{*}(M ; \mathbb{Z} / 2)$ for which there exists an algebraic model $(X, \varphi)$ of $M$ with $\varphi^{*}(A)=$ $H_{\text {alg }}^{*}(X ; \mathbb{Z} / 2)$ is wide open if $\operatorname{dim} M \geq 3$ (it is trivial if $\operatorname{dim} M \leq 1$, while its solution readily follows from [42, Corollary 1.12] for $M$ connected of dimension 2). The problem is unsolved even for $A$ contained in $A(M)$, when there are no obstructions to algebraic realizability of $A$. This paper provides partial solutions for a large class of subrings of $A(M)$ (cf. Theorems 1.1, 1.7, 2.10 and Corollaries 1.2, 1.4, 2.5, 1.8, 1.10).

The analogous problem of finding, for a fixed positive integer $r$, a characterization of the subgroups $G$ of $H^{r}(M ; \mathbb{Z} / 2)$ for which there exists an algebraic model $(X, \varphi)$ of $M$ with $\varphi^{*}(G)=H_{\text {alg }}^{r}(X ; \mathbb{Z} / 2)$ is more tractable. It is completely solved in [17, Theorems 1.2 and 1.3] and [42, Corollary 1.12] for $r=1$. The present paper contains a complete solution, under the assumption $\operatorname{dim} M \geq 5$, for $r=2$ (cf. Corollary 1.3) and several partial results for $r \geq 3$ (cf. Corollaries 1.6, 1.9 and 1.11). A necessary condition for the existence of such model $(X, \varphi)$ is that all cup products $w_{i_{1}}(M) \cup \cdots \cup w_{i_{p}}(M)$ be in $G$, where $i_{1}, \ldots, i_{p}$ are nonnegative integers with $i_{1}+\cdots+i_{p}=r$.

As the initial step, a suitable class of subrings of $H^{*}(M ; \mathbb{Z} / 2)$ will be defined.

If $h: M \rightarrow P$ is a smooth map into a compact smooth manifold $P$, then a standard transversality argument implies that $h^{*}(A(P)) \subseteq A(M)$ (cf. also [27, Proposition 2.15]). A subring $B$ of $H^{*}(M ; \mathbb{Z} / 2)$ is said to be full if $B=h^{*}\left(H^{*}(P ; \mathbb{Z} / 2)\right)$ for some $h: M \rightarrow P$ with $A(P)=H^{*}(P ; \mathbb{Z} / 2)$. Every full subring is contained in $A(M)$.

For any collection $F$ of real vector bundles on $M$, the subring $F(M)$ generated by the Stiefel-Whitney classes of the vector bundles in $F$ is a full subring of $H^{*}(M ; \mathbb{Z} / 2)$. Indeed, the collection $F$ can be assumed to be finite, the set $H^{*}(M ; \mathbb{Z} / 2)$ being finite, and hence, the assertion readily follows by making use of smooth classifying maps and Künneth's theorem (cf. [30,32,59]).

For any subring $B$ and any subset $T$ of $H^{*}(M ; \mathbb{Z} / 2)$, let $B[T]$ denote the extension of $B$ by $T$, that is, the subring of $H^{*}(M ; \mathbb{Z} / 2)$ generated by $B$ and $T$. A cohomology class in $H^{*}(M ; \mathbb{Z} / 2)$ will be called regular if it corresponds via the Poincaré duality 
to a homology class in $H_{*}(M ; \mathbb{Z} / 2)$ represented by a compact smooth submanifold of $M$. The subset $T$ will be called regular if each cohomology class in $T$ is regular. A subring of $H^{*}(M ; \mathbb{Z} / 2)$ that is the extension of a full subring by a regular subset is said to be admissible. An admissible subring $A$ is said to be $r$-admissible, where $r$ is a nonnegative integer, if it can be written as $A=B[T]$ for some full subring $B$ and some regular subset $T$, with $T$ disjoint from $H^{i}(M ; \mathbb{Z} / 2)$ for $0 \leq i \leq r-1$. Thus, admissible is the same as 0 -admissible. By a transversality argument, each admissible subring $A$ can be written as $A=F(M)[T]$, where $F$ is a finite collection of real vector bundles and $T$ is a regular subset. In particular, the definitions of an admissible subring used here and in [45] are equivalent. The largest admissible subring is $A(M)$. If $\operatorname{dim} M \leq 5$, then each cohomology class in $H^{*}(M ; \mathbb{Z} / 2)$ is regular [62, Théorème II.26], and hence, every subring of $H^{*}(M ; \mathbb{Z} / 2)$ is admissible.

Relationships between admissible subrings and $H_{\mathrm{alg}}^{*}(-; \mathbb{Z} / 2)$ are investigated below. The main results, whose proofs are postponed until Sect. 2, are Theorems 1.1 and 1.7. Their significance is elaborated upon in a series of corollaries. Some simple topological facts, contained in Proposition 1.12, are also required for the derivation of the corollaries.

As usual, the $i$ th Steenrod square operation will be denoted by $\mathrm{Sq}^{i}$. Only $\mathrm{Sq}^{1}$ is used in Sect. 1.

For any nonnegative integer $k$ and any subring $A$ of $H^{*}(M ; \mathbb{Z} / 2)$, let

$$
A^{k}:=A \cap H^{k}(M ; \mathbb{Z} / 2) .
$$

Theorem 1.1 Let $M$ be a compact connected smooth manifold and let $r$ be a positive integer with $2 r+1 \leq \operatorname{dim} M$. For an $r$-admissible subring $A$ of $H^{*}(M ; \mathbb{Z} / 2)$ with $A^{i}=0$ for $1 \leq i \leq r-2$, the following conditions are equivalent:

(a) There exists an algebraic model $(X, \varphi)$ of $M$ satisfying

$$
\varphi^{*}(A) \subseteq H^{*}(X ; \mathbb{Z} / 2) \text { and } \varphi^{*}\left(A^{k}\right)=H_{\mathrm{alg}}^{k}(X ; \mathbb{Z} / 2) \text { for } 0 \leq k \leq r
$$

(b) $w_{k}(M)$ is in $A^{k}$ for $0 \leq k \leq r$.

Of course, the condition $A^{i}=0$ for $1 \leq i \leq r-2$ is vacuous if $r=1$ or $r=2$. If $r=1$, then Theorem 1.1 is a minor improvement upon [17, Theorems 1.2 and 1.3]. The case $r=2$ is much more interesting.

Corollary 1.2 Let $M$ be a compact connected smooth manifold of dimension at least 5. For an admissible subring $A$ of $H^{*}(M ; \mathbb{Z} / 2)$, the following conditions are equivalent:

(a) There exists an algebraic model $(X, \varphi)$ of $M$ satisfying

$$
\varphi^{*}(A) \subseteq H_{\mathrm{alg}}^{*}(X ; \mathbb{Z} / 2) \text { and } \varphi^{*}\left(A^{k}\right)=H_{\mathrm{alg}}^{k}(X ; \mathbb{Z} / 2) \text { for } k=0,1,2 \text {. }
$$

(b) $w_{k}(M)$ is in $A^{k}$ for $k=0,1,2$.

Proof According to Proposition 1.12( $\left.\mathrm{p}_{1}\right)$, each admissible subring is 2-admissible, and hence, it suffices to apply Theorem 1.1 with $r=2$. 
Corollary 1.2 was proved in [45] for $M$ with homology group $H_{m-2}(M ; \mathbb{Z})$ having no 2-torsion, where $m=\operatorname{dim} M$. This additional assumption removed the main difficulty in the proof.

It is interesting to extract from Corollary 1.2 and previously known results information on the behavior of $H_{\mathrm{alg}}^{2}(-; \mathbb{Z} / 2)$. Let $A^{r}(M):=A(M)^{r}$. According to [20,61], for any compact smooth manifold $M$, the group $A^{2}(M)$ can be described as follows: $A^{2}(M)=W^{2}(M)$, where

$$
\begin{aligned}
W^{2}(M):=\left\{v \in H^{2}(M ; \mathbb{Z} / 2) \mid v=\right. & w_{2}(\xi) \text { for some real vector } \\
& \text { bundle } \left.\xi \text { on } M \text { with } w_{1}(\xi)=0\right\}
\end{aligned}
$$

and $w_{k}(\xi)$ denotes the $k$ th Stiefel-Whitney class of $\xi$ for $k \geq 0$. Thus, $W^{2}(M)=$ $H^{2}(M ; \mathbb{Z} / 2)$ if $\operatorname{dim} M \leq 5$. However, for each integer $n \geq 6$, there exists an $n$-dimensional compact connected smooth manifold $N$ with $W^{2}(N) \neq H^{2}(N, \mathbb{Z} / 2)$ [61]. On the other hand,

$$
H_{\mathrm{alg}}^{2}(X ; \mathbb{Z} / 2) \subseteq W^{2}(X)
$$

for every compact nonsingular real algebraic set $X$ (cf. $[12,18]$ and, for an elementary topological proof, [23]). In particular, $H_{\text {alg }}^{2}(Y ; \mathbb{Z} / 2) \neq H^{2}(Y ; \mathbb{Z} / 2)$ for every algebraic model $Y$ of $N$.

Corollary 1.3 Let $M$ be a compact connected smooth manifold of dimension at least 5. For a subgroup $G$ of $H^{2}(M ; \mathbb{Z} / 2)$, the following conditions are equivalent:

(a) There exists an algebraic model $(X, \varphi)$ of $M$ satisfying

$$
\varphi^{*}(G)=H_{\mathrm{alg}}^{2}(X ; \mathbb{Z} / 2)
$$

(b) $w_{1}(M) \cup w_{1}(M)$ and $w_{2}(M)$ are in $G$, and $G \subseteq W^{2}(M)$.

Proof If (a) holds, then $w_{1}(X) \cup w_{1}(X)$ and $w_{2}(X)$ belong to $\varphi^{*}(G)$, and $\varphi^{*}(G) \subseteq$ $W^{2}(X)$. Hence, (b) follows.

Suppose that (b) holds. For each cohomology class $v$ in $W^{2}(M)$, let $\xi_{v}$ be a real vector bundle on $M$ with $w_{1}\left(\xi_{v}\right)=0$ and $w_{2}\left(\xi_{v}\right)=v$. Let $F$ be the collection consisting of the tangent bundle to $M$ and the $\xi_{v}$ for $v$ in $G$. The subring $A:=F(M)$ of $H^{*}(M, \mathbb{Z} / 2)$ is admissible, $A^{2}=G$, and $w_{i}(M)$ is in $A^{i}$ for $i \geq 0$. Hence, Corollary 1.2 implies that (a) is satisfied.

Corollary 1.3 was already conjectured in [20], but proved there only for $M$ orientable, that is, $w_{1}(M)=0$. In [40], Corollary 1.3 was proved under very restrictive assumptions on the group $H^{m-2}(M ; \mathbb{Z} / 2)$, where $m=\operatorname{dim} M$. The methods used in $[20,40]$ do not work without these extra hypotheses.

There is also a version of Corollary 1.2 for an arbitrary, not necessarily admissible, subring.

Corollary 1.4 Let $M$ be a compact connected smooth manifold of dimension at least 5. For a subring $A$ of $H^{*}(M ; \mathbb{Z} / 2)$, the following conditions are equivalent: 
(a) There exists an algebraic model $(X, \varphi)$ of $M$ satisfying

$$
\varphi^{*}\left(A^{k}\right)=H_{\mathrm{alg}}^{k}(X ; \mathbb{Z} / 2) \text { for } k=0,1,2 .
$$

(b) $w_{k}(M)$ is in $A^{k}$ for $k=0,1,2$, and $A^{2} \subseteq W^{2}(M)$.

Proof It is already explained that (a) implies (b).

Suppose now that (b) holds. Each cohomology class $u$ in $H^{1}(M ; \mathbb{Z} / 2)$ can be written as $u=w_{1}\left(\gamma_{u}\right)$ for some real line bundle $\gamma_{u}$ on $M$. Similarly, each cohomology class $v$ in $W^{2}(M)$ can be written as $v=w_{2}\left(\xi_{v}\right)$ for some real vector bundle $\xi_{v}$ on $M$ with $w_{1}\left(\xi_{v}\right)=0$. Let $F$ be the collection consisting of $\gamma_{u}$ for $u$ in $A^{1}, \xi_{v}$ for $v$ in $A^{2}$ and the tangent bundle to $M$. The subring $C:=F(M)$ of $H^{*}(M ; \mathbb{Z} / 2)$ is admissible with $C^{k}=A^{k}$ for $k=0,1,2$. Corollary 1.2 applied to the subring $C$ implies (a).

Theorem 1.1 with $r=3$ implies the following:

Corollary 1.5 Let $M$ be a compact connected orientable smooth manifold of dimension at least 7. For an admissible subring $A$ of $H^{*}(M ; \mathbb{Z} / 2)$ with $A^{1}=0$, the following conditions are equivalent:

(a) There exists an algebraic model $(X, \varphi)$ of $M$ satisfying

$$
\varphi^{*}(A) \subseteq H_{\mathrm{alg}}^{*}(X ; \mathbb{Z} / 2) \text { and } \varphi^{*}\left(A^{k}\right)=H_{\mathrm{alg}}^{k}(X ; \mathbb{Z} / 2) \text { for } k=0,1,2,3 \text {. }
$$

(b) $w_{i}(M)$ is in $A^{i}$ for $i=2,3$, and $\mathrm{Sq}^{1}\left(A^{2}\right) \subseteq A^{3}$.

Proof According to $\left[4\right.$, Theorem 6.6], $\mathrm{Sq}^{1}\left(H_{\text {alg }}^{2}(-; \mathbb{Z} / 2)\right) \subseteq H_{\text {alg }}^{3}(-; \mathbb{Z} / 2)$, and therefore, (a) implies (b).

Suppose now that (b) holds. By Proposition 1.12( $\left.\mathrm{p}_{2}\right)$, there exists a 3-admissible subring $\bar{A}$ of $H^{*}(M ; \mathbb{Z} / 2)$ such that $A \subseteq \bar{A}$ and $A^{k}=\bar{A}^{k}$ for $k=0,1,2,3$. The orientability of $M$ implies $w_{1}(M)=0$. Hence, (a) follows by applying Theorem 1.1 with $r=3$ to the subring $\bar{A}$.

It would be interesting, but very hard, to extend Corollary 1.3 to subgroups of $H^{r}(M ; \mathbb{Z} / 2)$ with $r \geq 3$. The following partial result is available.

Corollary 1.6 Let $M$ be a compact connected smooth manifold and let $r \geq 3$ be an integer with $2 r+1 \leq \operatorname{dim} M$. Assume that $w_{i}(M)=0$ for $1 \leq i \leq r-2$. If $G$ is a subgroup of $H^{r}(M ; \mathbb{Z} / 2)$ generated by some regular cohomology classes and $w_{r}(M)$, then there exists an algebraic model $(X, \varphi)$ of $M$ satisfying $\varphi^{*}(G)=H_{\mathrm{alg}}^{r}(X ; \mathbb{Z} / 2)$.

Proof The subring $A$ of $H^{*}(M ; \mathbb{Z} / 2)$ generated by $G$ and the cohomology classes $w_{k}(M)$ for $k \geq 0$ is $r$-admissible. Moreover, $A^{i}=0$ for $1 \leq i \leq r-2$ and $A^{r}=G$. It remains to apply Theorem 1.1 .

If $r=3$ in Corollary 1.6, then the condition $w_{i}(M)=0$ for $1 \leq i \leq r-2$ is equivalent to the orientability of $M$. 
Theorem 1.7 Let $M$ be a compact connected smooth manifold whose homology group $H_{r-1}(M ; \mathbb{Z})$ has no 2-torsion for some integer $r \geq 3$ with $2 r+1 \leq \operatorname{dim} M$. Let $A$ be an $r$-admissible subring of $H^{*}(M ; \mathbb{Z} / 2)$ with $A^{i}=0$ for $1 \leq i \leq r-4$. Assume that $w_{j}(M)$ is in $A^{j}$ for $0 \leq j \leq r$. Then, there exists an algebraic model $(X, \varphi)$ of $M$ satisfying

$$
\begin{aligned}
& \varphi^{*}(A) \subseteq H_{\mathrm{alg}}^{*}(X ; \mathbb{Z} / 2) \text { and } \\
& \varphi^{*}\left(A^{k}\right)=H_{\mathrm{alg}}^{k}(X ; \mathbb{Z} / 2) \text { for } k \in\{0,1, \ldots, r-2, r\} \cup\{2\} .
\end{aligned}
$$

Moreover, the last equality holds for $0 \leq k \leq r$ if $r \geq 4$, and either the homology group $H_{r-2}(M ; \mathbb{Z})$ has no 2-torsion or $\bar{A}^{r-3}=0$.

Clearly, the condition $A^{i}=0$ for $1 \leq i \leq r-4$ is vacuous if $r=3$ or $r=4$. The case $r=3$ is of particular interest.

Corollary 1.8 Let $M$ be a compact connected smooth manifold of dimension at least 7 , whose homology group $\mathrm{H}_{2}(M ; \mathbb{Z})$ has no 2-torsion. For an admissible subring $A$ of $H^{*}(M ; \mathbb{Z} / 2)$, the following conditions are equivalent:

(a) There exists an algebraic model $(X, \varphi)$ of $M$ satisfying

$$
\varphi^{*}(A) \subseteq H_{\mathrm{alg}}^{*}(X ; \mathbb{Z} / 2) \text { and } \varphi^{*}\left(A^{k}\right)=H_{\mathrm{alg}}^{k}(X ; \mathbb{Z} / 2) \text { for } k=0,1,2,3 \text {. }
$$

(b) $w_{k}(M)$ is in $A^{k}$ for $k=0,1,2,3$.

Proof It suffices to prove that (b) implies (a). According to Proposition 1.12( $\left.\mathrm{p}_{3}\right)$, the subring $A$ is 3-admissible, and hence, it suffices to apply Theorem 1.7 with $r=3$.

A much weaker version of Corollary 1.8 was proved in [45] for a spin manifold $M$ whose homology group $H_{i}(M ; \mathbb{Z})$ has no 2 -torsion for $i=1,2$. By definition, $M$ is a spin manifold if $w_{1}(M)=0$ and $w_{2}(M)=0$, which automatically implies $w_{3}(M)=0$ (cf. [52, p. 94]).

Corollary 1.9 Let $M$ be a compact connected smooth manifold of dimension at least 7 , whose homology group $\mathrm{H}_{2}(M ; \mathbb{Z})$ has no 2-torsion. For an admissible subring $A$ of $H^{*}(M ; \mathbb{Z} / 2)$, the following conditions are equivalent:

(a) There exists an algebraic model $(X, \varphi)$ of $M$ satisfying

$$
\varphi^{*}\left(A^{3}\right)=H_{\text {alg }}^{3}(X ; \mathbb{Z} / 2) .
$$

(b) $w_{1}(M) \cup w_{1}(M) \cup w_{1}(M), w_{1}(M) \cup w_{2}(M)$ and $w_{3}(M)$ are in $A^{3}$.

Proof It is already known that (a) implies (b).

Suppose now that (b) holds. According to Proposition $1.12\left(\mathrm{p}_{3}\right)$, the subgroup $A^{3}$ of $H^{3}(M ; \mathbb{Z} / 2)$ is generated by regular cohomology classes. Hence, the subring $C$ of $H^{*}(M ; \mathbb{Z} / 2)$ generated by $A^{3}$ and $w_{i}(M)$ for $i \geq 0$ is admissible and $C^{3}=A^{3}$. Condition (a) follows by applying Corollary 1.8 to the subring $C$.

Theorem 1.7 with $r=4$ takes the following form: 
Corollary 1.10 Let $M$ be a compact connected smooth manifold of dimension at least 9 , whose homology group $\mathrm{H}_{3}(M ; \mathbb{Z})$ has no 2-torsion. For a 4-admissible subring $A$ of $H^{*}(M ; \mathbb{Z} / 2)$, the following conditions are equivalent:

(a) There exists an algebraic model $(X, \varphi)$ of $M$ satisfying

$$
\varphi^{*}(A) \subseteq H_{\mathrm{alg}}^{k}(X ; \mathbb{Z} / 2) \text { and } \varphi^{*}\left(A^{k}\right)=H_{\mathrm{alg}}^{k}(X ; \mathbb{Z} / 2) \text { for } k=0,1,2,4 \text {. }
$$

(b) $\quad w_{j}(M)$ is in $A^{j}$ for $j=0,1,2,3,4$.

Moreover, $k=3$ can be added in condition (a) if either the homology group $\mathrm{H}_{2}(M ; \mathbb{Z})$ has no 2-torsion or $A^{1}=0$.

Proof Since the ring $A$ is 4-admissible, it readily follows that $\operatorname{Sq}^{1}\left(A^{2}\right) \subseteq A^{3}$. By Wu's formula [52, p. 94], $\mathrm{Sq}^{1}\left(w_{2}(M)\right)=w_{1}(M) \cup w_{2}(M)+w_{3}(M)$. Consequently, if $w_{j}(M)$ is in $A^{j}$ for $j=1,2$, then $w_{3}(M)$ is in $A^{3}$. If (a) holds, then $w_{j}(M)$ is in $A^{j}$ for $j=0,1,2,4$, and hence, (b) is satisfied. According to Theorem 1.7 with $r=4$, condition (b) implies (a).

It is an open problem whether or not Corollary 1.10 remains true if the homology group $H_{i}(M ; \mathbb{Z})$ has no 2-torsion for $i=2,3$ and the subring $A$ is admissible, but not necessarily 4-admissible. No result similar to Corollary 1.10 is available in the literature.

Under an additional assumption on $M$, Corollary 1.6 can be strengthened as follows.

Corollary 1.11 Let $M$ be a compact connected smooth manifold whose homology group $H_{r-1}(M ; \mathbb{Z})$ has no 2-torsion for some integer $r \geq 3$ with $2 r+1 \leq \operatorname{dim} M$. Let $G$ be a subgroup of $H^{r}(M ; \mathbb{Z} / 2)$ generated by some regular cohomology classes and all cup products $w_{i_{1}}(M) \cup \cdots \cup w_{i_{p}}(M)$, where $i_{1}, \ldots i_{p}$ are nonnegative integers with $i_{1}+\cdots+i_{p}=r$. If $w_{i}(M)=0$ for $1 \leq i \leq r-4$, then there exists an algebraic model $(X, \varphi)$ of $M$ satisfying $\varphi^{*}(G)=H_{\mathrm{alg}}(X ; \mathbb{Z} / 2)$.

Proof The subring $A$ of $H^{*}(M ; \mathbb{Z} / 2)$ generated by $G$ and $w_{j}(M)$ for $j \geq 0$ is $r$-admissible, $A^{i}=0$ for $1 \leq i \leq r-4$, and $A^{r}=G$. Hence, it suffices to apply Theorem 1.7.

In Corollary 1.11, the condition $w_{i}(M)=0$ for $1 \leq i \leq r-4$ is vacuous if $r=3$ or $r=4$, while it is equivalent to the orientability of $M$ if $r=5$. It follows from Proposition $1.12\left(\mathrm{p}_{3}\right)$ that Corollary 1.9 is equivalent to Corollary 1.11 with $r=3$.

The properties of admissible rings used in the proofs of the corollaries above are contained in the following:

Proposition 1.12 Let $M$ be a compact connected smooth manifold. Any admissible subring $M$ of $H^{*}(M ; \mathbb{Z} / 2)$ has the following properties:

$\left(p_{1}\right)$ A is 2-admissible.

$\left(p_{2}\right)$ If $\operatorname{Sq}^{1}\left(A^{2}\right) \subseteq A^{3}$, then there exists a 3 -admissible subring $\bar{A}$ of $H^{*}(M ; \mathbb{Z} / 2)$ satisfying $A \subseteq \bar{A}$ and $A^{i}=\bar{A}^{i}$ for $i=0,1,2,3$.

$\left(p_{3}\right)$ If the homology group $\mathrm{H}_{2}(M ; \mathbb{Z})$ has no 2-torsion, then $A$ is 3-admissible and the subgroup $A^{3}$ of $H^{3}(M ; \mathbb{Z} / 2)$ is generated by regular cohomology classes. 
Proof By Künneth's theorem, each subring of $H^{*}(M ; \mathbb{Z} / 2)$ that is generated by two full subrings is also full.

The admissible subring $A$ can be written as $A=B[T]$, where $B$ is a full subring and $T$ is a regular subset of $H^{*}(M ; \mathbb{Z} / 2)$. Let $T^{i}:=T \cap H^{i}(M ; \mathbb{Z} / 2)$ for $i \geq 0$. One has $A^{0}=B^{0}=H^{0}(M ; \mathbb{Z} / 2)$, the manifold $M$ being connected, and hence, it can be assumed that $T^{0}=\varnothing$.

For each cohomology class $u$ in $H^{1}(M ; \mathbb{Z} / 2)$, let $\gamma_{u}$ be a real line bundle on $M$ with $w_{1}\left(\gamma_{u}\right)=u$. Let $F_{1}:=\left\{\gamma_{u} \mid u \in T^{1}\right\}$. The subring $B\left(F_{1}\right)$ of $H^{*}(M ; \mathbb{Z} / 2)$ generated by $B$ and $F_{1}(M)$ is full. Property $\left(p_{1}\right)$ follows since $A=B\left(F_{1}\right)\left[T \backslash T^{1}\right]$.

According to Wu's formula [52, p. 94], for each real vector bundle $\xi$ on $M$,

$$
\operatorname{Sq}^{1}\left(w_{2}(\xi)\right)=w_{1}(\xi) \cup w_{2}(\xi)+w_{3}(\xi)
$$

For each cohomology class $v$ in $W^{2}(M)$, let $\xi_{v}$ be a real vector bundle on $M$ with $w_{1}\left(\xi_{v}\right)=0$ and $w_{2}\left(\xi_{v}\right)=v$. The admissibility of $A$ implies that $A^{2}$ is contained in $A^{2}(M)=W^{2}(M)$. In particular, the set $F_{2}:=\left\{\xi_{v} \mid v \in T^{2}\right\}$ is well defined. The subring $B\left(F_{1}, F_{2}\right)$ of $H^{*}(M ; \mathbb{Z} / 2)$ generated by $B$ and $\left(F_{1} \cup F_{2}\right)(M)$ is full, and the subring $\bar{A}:=B\left(F_{1}, F_{2}\right)\left[T \backslash\left(T^{1} \cup T^{2}\right)\right]$ is 3-admissible. Moreover, $A \subseteq \bar{A}$ and $A^{i}=\bar{A}^{i}$ for $i=0,1,2$. If $\operatorname{Sq}^{1}\left(A^{2}\right) \subseteq A^{3}$, then $(*)$ with $\xi=\xi_{v}$ implies that $w_{3}\left(\xi_{v}\right)=\operatorname{Sq}^{1}(v)$ is in $A^{3}$ for $v$ in $T^{2}$. Consequently, $A^{3}=\bar{A}^{3}$. Property $\left(\mathrm{p}_{2}\right)$ is proved.

Suppose now that the homology group $H_{2}(M ; \mathbb{Z})$ has no 2-torsion. According to the universal coefficient theorem, the cohomology group $H^{3}(M ; \mathbb{Z})$ has no 2-torsion and the reduction modulo 2 homomorphism $\rho: H^{2}(M ; \mathbb{Z}) \rightarrow H^{2}(M ; \mathbb{Z} / 2)$ is surjective. For each cohomology class $z$ in $H^{2}(M ; \mathbb{Z})$, let $\lambda_{z}$ be a complex line bundle on $M$ whose first Chern class is $z$. Regarding $\lambda_{z}$ as a rank 2 real vector bundle, one gets $w_{1}\left(\lambda_{z}\right)=0$ and $w_{2}\left(\lambda_{z}\right)=\rho(z)$. Consequently, $W^{2}(M)=H^{2}(M ; \mathbb{Z} / 2)$, and it can be assumed that for each $v$ in $H^{2}(M ; \mathbb{Z} / 2)$, the vector bundle $\xi_{v}$ above is of rank 2 . In particular, $w_{j}\left(\xi_{v}\right)=0$ for $j \geq 3$. It follows that then $A$ is equal to the subring $\bar{A}$ constructed above, and hence, $A$ is 3 -admissible. It remains to prove that $A^{3}$ is generated by regular cohomology classes. Each cohomology class in $H^{1}(M, \mathbb{Z} / 2)$ is regular. Similarly, each cohomology class $v$ in $H^{2}(M ; \mathbb{Z} / 2)$ is regular since it is Poincaré dual to the homology class represented by the zero locus of an arbitrary smooth section of $\xi_{v}$ that is transverse to the zero section (cf. [27, Proposition 2.15]). The homomorphism $\mathrm{Sq}^{1}: H^{2}(M ; \mathbb{Z} / 2) \rightarrow H^{3}(M ; \mathbb{Z} / 2)$ is zero, the homomorphism $\rho$ being surjective [52, p. 182], and hence, $(*)$ gives $w_{3}(\xi)=w_{1}(\xi) \cup w_{2}(\xi)$. The proof is complete since cup product of regular cohomology classes is a regular class.

Convention Henceforth, smooth submanifolds are assumed to be closed subsets of the ambient manifold.

\section{Proofs and further results}

The language of real algebraic geometry, as in [16], is used throughout this section. The term real algebraic variety designates a locally ringed space isomorphic to an algebraic subset of $\mathbb{R}^{n}$, for some $n$, endowed with the Zariski topology and the sheaf 
of real-valued regular functions (such objects are called affine real algebraic varieties in [16]). The Grassmannian $\mathbb{G}_{n, r}(\mathbb{R})$ of $r$-dimensional vector subspaces of $\mathbb{R}^{n}$ is a real algebraic variety in this sense [16, Theorem 3.4.4]. Morphisms between real algebraic varieties are called regular maps. Every real algebraic variety carries also the Euclidean topology, which is induced by the usual matric on $\mathbb{R}$. Unless explicitly stated otherwise, all topological notions relating to real algebraic varieties refer to the Euclidean topology.

A topological real vector bundle on a real algebraic variety $X$ is said to admit an algebraic structure if it is isomorphic to an algebraic subbundle of the trivial vector bundle on $X$ with total space $X \times \mathbb{R}^{p}$ for some $p$.

For any smooth manifolds $N$ and $P$, the space of smooth maps $\mathcal{C}^{\infty}(N, P)$ is endowed with the $\mathcal{C}^{\infty}$ topology [30]. The source manifold will always be assumed to be compact, and hence, the weak $\mathcal{C}^{\infty}$ topology coincides with the strong one. The unoriented bordism group of $P$ is denoted by $\mathfrak{N}_{*}(P)$. If $W$ is a nonsingular real algebraic variety, then a bordism class in $\mathfrak{N}_{*}(W)$ is said to be algebraic, provided that it can be represented by a regular map from a compact nonsingular real algebraic variety into $W$. The set $\mathfrak{N}_{*}^{\text {alg }}(W)$ of all algebraic bordism classes in $\mathfrak{N}_{*}(W)$ forms a subgroup.

The main approximation theorem of real algebraic geometry, in the form most suitable for this paper, will be recalled first. It is just a reformulation of very similar results proved in $[1,8,13,14,64]$.

Theorem 2.1 (cf. [42, Theorem 4.4]) Let $M$ be a compact smooth submanifold of $\mathbb{R}^{n}$ and let $W$ be a nonsingular real algebraic variety. Let $f: M \rightarrow W$ be a smooth map whose bordism class in $\mathfrak{N}_{*}(W)$ is algebraic. Suppose that $M$ contains a (possibly empty) subset $Z$ which is the union of finitely many nonsingular algebraic subsets of $\mathbb{R}^{n},\left.f\right|_{Z}: Z \rightarrow W$ is a regular map, and the restriction to $Z$ of the tangent bundle of $M$ admits an algebraic structure. If $2 \operatorname{dim} M+1 \leq n$, then there exists a smooth embedding $e: M \rightarrow \mathbb{R}^{n}$, a nonsingular algebraic subset $X$ of $\mathbb{R}^{n}$, and a regular map $g: X \rightarrow W$ such that $X=e(M), Z \subseteq X,\left.e\right|_{Z}: Z \rightarrow \mathbb{R}^{n}$ is the inclusion map, $\left.g\right|_{Z}=\left.f\right|_{Z}$, and $g \circ \bar{e}$ is homotopic to $f$, where $\bar{e}: M \rightarrow X$ is the smooth diffeomorphism defined by $\bar{e}(x)=e(x)$ for all $x$ in $M$. Furthermore, given a neighborhood $\mathcal{U}$ of the inclusion map $M \hookrightarrow \mathbb{R}^{n}$ in the space $\mathcal{C}^{\infty}\left(M, \mathbb{R}^{n}\right)$ and a neighborhood $\mathcal{V}$ of $f$ in $\mathcal{C}^{\infty}(M, W)$, the objects $e, X, g$ can be chosen in such a way that e is in $\mathcal{U}$ and $g \circ \bar{e}$ is in $\mathcal{V}$.

In favorable situations, the bordism condition in Theorem 2.1 is automatically satisfied.

Proposition 2.2 Let $V$ and $W$ be compact nonsingular real algebraic varieties. Then:

(i) $\mathfrak{N}_{*}^{\text {alg }}(V)=\mathfrak{N}_{*}(V)$ if and only if $H_{*}^{\text {alg }}(V ; \mathbb{Z} / 2)=H_{*}(V ; \mathbb{Z} / 2)$.

(ii) The equality $H_{*}^{\text {alg }}(V \times W ; \mathbb{Z} / 2)=H_{*}(V \times W ; \mathbb{Z})$ holds, provided that $H_{*}^{\text {alg }}(V ; \mathbb{Z} / 2)=H_{*}(V ; \mathbb{Z} / 2)$ and $H_{*}^{\text {alg }}(W ; \mathbb{Z} / 2)=H_{*}(W ; \mathbb{Z} / 2)$.

Moreover, $H_{*}^{\text {alg }}\left(\mathbb{G}_{n, r}(\mathbb{R}) ; \mathbb{Z} / 2\right)=H_{*}\left(\mathbb{G}_{n, r}(\mathbb{R}) ; \mathbb{Z} / 2\right)$.

Proof Condition (i) is a consequence of deep results from topology (cf. [8, Lemma 2.7.1]). Condition (ii) follows from Künneth's theorem. The last assertion is a standard fact (cf. [16, Proposition 11.3.3]). 
The result that will be recalled next is used in constructions of nonalgebraic cohomology classes. For any compact nonsingular real algebraic variety $X$, let $\operatorname{Alg}^{k}(X)$ denote the set of all elements $u$ in $H^{k}(X ; \mathbb{Z} / 2)$ for which there exist a compact nonsingular irreducible real algebraic variety $T$, two points $t_{0}$ and $t_{1}$ in $T$ and the cohomology class $z$ in $H_{\text {alg }}^{k}(X \times T ; \mathbb{Z} / 2)$ such that

$$
u=i_{t_{1}}^{*}(z)-i_{t_{0}}^{*}(z)
$$

where $i_{t}: X \rightarrow X \times T$ is defined by $i_{t}(x)=(x, t)$ for $t \in T$ and $x \in X$. An equivalent description of $\operatorname{Alg}^{k}(X)$, which immediately implies that $\operatorname{Alg}^{k}(X)$ is a subgroup of $H_{\mathrm{alg}}^{k}(X ; \mathbb{Z} / 2)$, is given in $[38,40]$. The groups $H_{\mathrm{alg}}^{k}(-; \mathbb{Z} / 2)$ and $\operatorname{Alg}^{k}(-)$ have the expected functorial property. If $f: X \rightarrow Y$ is a regular map between compact nonsingular real algebraic varieties, then the induced homomorphism $f^{*}: H^{*}(Y ; \mathbb{Z} / 2) \rightarrow$ $H^{*}(X ; \mathbb{Z} / 2)$ satisfies

$$
f^{*}\left(H_{\mathrm{alg}}^{k}(Y ; \mathbb{Z} / 2)\right) \subseteq H_{\mathrm{alg}}^{k}(X ; \mathbb{Z} / 2) \text { and } f^{*}\left(\operatorname{Alg}^{k}(Y)\right) \subseteq \operatorname{Alg}^{k}(X)
$$

(cf. [27, Section 5] or [4, 15] for the former inclusion and [40] for the latter).

Example 2.3 Let $\Sigma$ be an irreducible nonsingular real algebraic variety with precisely two connected components $\Sigma_{0}$ and $\Sigma_{1}$, each diffeomorphic to the unit circle. For example, one can take

$$
\Sigma=\left\{\left(x_{1}, x_{2}\right) \in \mathbb{R}^{2} \mid x_{1}^{4}-4 x_{1}^{2}+x_{2}^{2}+1=0\right\}
$$

Let $z$ be the cohomology class in $H^{1}(\Sigma \times \Sigma ; \mathbb{Z} / 2)$ that is Poincare dual to the homology class in $H_{1}(\Sigma \times \Sigma ; \mathbb{Z} / 2)$ represented by the diagonal of $\Sigma \times \Sigma$. For any point $t$ in $\Sigma$, let $i_{t}: \Sigma \rightarrow \Sigma \times \Sigma$ be defined by $i_{t}(x)=(x, t)$ for all $x$ in $\Sigma$. The cohomology class $i_{t}^{*}(z)$ in $H^{1}(\Sigma ; \mathbb{Z} / 2)$ is Poincaré dual to the homology class in $H_{1}(\Sigma ; \mathbb{Z} / 2)$ represented by the point $t$. Let $t_{j}$ be a point in $\Sigma_{j}$ for $j=0,1$. The cohomology class $u:=i_{t_{1}}^{*}(z)-i_{t_{0}}^{*}(z)$ is in $\operatorname{Alg}^{1}(\Sigma)$. If $\sigma: \Sigma_{0} \hookrightarrow \Sigma$ is the inclusion map, then $\sigma^{*}(u)$ generates $H^{1}\left(\Sigma_{0} ; \mathbb{Z} / 2\right) \cong \mathbb{Z} / 2$ and hence

$$
H^{1}\left(\Sigma_{0} ; \mathbb{Z} / 2\right)=\sigma^{*}\left(H^{1}(\Sigma ; \mathbb{Z} / 2)\right)=\sigma^{*}\left(\operatorname{Alg}^{1}(\Sigma)\right)
$$

Consequently, the functoriality of $\operatorname{Alg}^{1}(-)$ implies that

$$
r^{*}\left(H^{1}(\Sigma ; \mathbb{Z} / 2)\right) \subseteq \operatorname{Alg}^{1}(Y)
$$

for every nonsingular real algebraic variety $Y$ and every regular map $r: Y \rightarrow \Sigma$ with $r(Y) \subseteq \Sigma_{0}$.

As usual, the Kronecker index (scalar product) of cohomology and homology classes will be denoted by $\langle-,-\rangle$. For any $m$-dimensional compact smooth manifold $M$, let $[M]$ denote its fundamental class in $H_{m}(M ; \mathbb{Z} / 2)$. 
Theorem 2.4 (cf. [38, Theorem 2.1]) Let $X$ be a compact nonsingular real algebraic variety. Then, $\langle u \cup v,[X]\rangle=0$ for all $u$ in $H_{\mathrm{alg}}^{k}(X ; \mathbb{Z} / 2)$ and $v$ in $\operatorname{Alg}^{l}(X)$, where $k+l=\operatorname{dim} X$.

If $K$ is a $k$-dimensional smooth submanifold of $M$, let $[K]_{M}$ denote the homology class in $H_{k}(M ; \mathbb{Z} / 2)$ represented by $K$, that is, $[K]_{M}:=\kappa_{*}([K])$, where $\kappa: K \hookrightarrow M$ is the inclusion map. The unit 1 -sphere and the unit 1 -disk will be denoted by $\mathbb{S}^{1}$ and $\mathbb{D}^{1}$, respectively.

The following technical result will be very useful.

Lemma 2.5 Let L be a $(k+1)$-dimensional compact smooth submanifold of $\mathbb{R}^{n}$ and let $K$ be a $k$-dimensional smooth submanifold of $L$ such that there is a smooth diffeomorphism $\theta: K \times \mathbb{S}^{1} \rightarrow L$ satisfying $\theta\left(K \times\left\{z_{0}\right\}\right)=K$ for some point $z_{0}$ in $\mathbb{S}^{1}$. Let $f: L \rightarrow V$ be a smooth map into a nonsingular real algebraic variety $V$. Let $\mathcal{U}$ be a neighborhood of the inclusion map $L \hookrightarrow \mathbb{R}^{n}$ in the space $\mathcal{C}^{\infty}\left(L, \mathbb{R}^{n}\right)$ and let $\mathcal{V}$ be a neighborhood of $f$ in $\mathcal{C}^{\infty}(L, V)$. Assume that $2 k+3 \leq n$, the map $f \circ \theta: K \times \mathbb{S}^{1} \rightarrow V$ has a continuous extension $K \times \mathbb{D}^{1} \rightarrow V$, and the bordism class of the map $\left.f\right|_{K}: K \rightarrow V$ in the group $\mathfrak{N}_{*}(V)$ is 0 . Then, there exists a smooth embedding $\varepsilon: L \rightarrow \mathbb{R}^{n}$, a nonsingular algebraic subset $Y$ of $\mathbb{R}^{n}$, and a regular map $g: Y \rightarrow V$ such that $Y=\varepsilon(L), \varepsilon$ is in $\mathcal{U}, g \circ \bar{\varepsilon}$ is in $\mathcal{V}$, and

$$
H_{\mathrm{alg}}^{k}(Y ; \mathbb{Z} / 2) \subseteq\left\{w \in H^{k}(Y ; \mathbb{Z} / 2) \mid\left\langle w, \bar{\varepsilon}_{*}\left([K]_{L}\right)\right\rangle=0\right\},
$$

where $\bar{\varepsilon}: L \rightarrow Y$ is the smooth diffeomorphism determined by $\varepsilon$.

Proof Let $\Sigma$ be as in Example 2.3, and let $h_{0}: \mathbb{S}^{1} \rightarrow \Sigma$ be a smooth embedding onto $\Sigma_{0}$. If $f_{0}: K \rightarrow V$ is defined by $f_{0}(x)=f\left(\theta\left(x, z_{0}\right)\right)$ for all $x$ in $K$, then the bordism class of $f_{0} \times h_{0}: K \times \mathbb{S}^{1} \rightarrow V \times \Sigma$ in the group $\mathfrak{N}_{*}(V \times \Sigma)$ is 0 . Indeed, this assertion follows since the bordism classes of $f_{0}: K \rightarrow V$ and $\left.f\right|_{K}: K \rightarrow V$ in $\mathfrak{N}_{*}(V)$ are equal, and the latter class is 0 by assumption.

If $F: K \times \mathbb{D}^{1} \rightarrow V$ is a continuous extension of $f \circ \theta: K \times \mathbb{S}^{1} \rightarrow V$, then the $\operatorname{map} H: K \times \mathbb{S}^{1} \times[0,1] \rightarrow V$,

$$
H(x, z, t)=F\left(x,(1-t) z+t z_{0}\right) \text { for }(x, z, t) \text { in } K \times \mathbb{S}^{1} \times[0,1],
$$

is a homotopy from $f \circ \theta$ to $f_{0} \circ \pi$, where $\pi: K \times \mathbb{S}^{1} \rightarrow K$ is the canonical projection. Hence, if $\rho: K \times \mathbb{S}^{1} \rightarrow \mathbb{S}^{1}$ is the canonical projection and $h:=h_{0} \circ \rho \circ \theta^{-1}$, the map

$$
(f, h) \circ \theta=(f \circ \theta, h \circ \theta): K \times \mathbb{S}^{1} \rightarrow V \times \Sigma
$$

is homotopic to

$$
\left(f_{0} \circ \pi, h_{0} \circ \rho\right)=f_{0} \times h_{0}: K \times \mathbb{S}^{1} \rightarrow V \times \Sigma .
$$

Consequently, the bordism class of $(f, h): L \rightarrow V \times \Sigma$ in $\mathfrak{N}_{*}(V \times \Sigma)$ is 0 . 
By Theorem 2.1 (with $M=L, Z=\emptyset$, and $W=V \times \Sigma$ ), there exist a smooth embedding $\varepsilon: L \rightarrow \mathbb{R}^{n}$, a nonsingular algebraic subset $Y$ of $\mathbb{R}^{n}$, and a regular map $(h, r): Y \rightarrow V \times \Sigma$ such that $Y=\varepsilon(L), \varepsilon$ is in $\mathcal{U}$, and $(g, r) \circ \bar{\varepsilon}$ is close to $(f, h)$ in $\mathcal{C}^{\infty}(L, V \times \Sigma)$, where $\bar{\varepsilon}: L \rightarrow Y$ is the smooth diffeomorphism determined by $\varepsilon$. In particular, $g \circ \bar{\varepsilon}$ is in $\mathcal{V}$, and $r$ is homotopic to $h \circ \bar{\varepsilon}^{-1}$. The proof can be completed as follows. Let $v$ be the cohomology class in $H^{1}(\Sigma ; \mathbb{Z} / 2)$ that is Poincaré dual to the homology class represented by the point $y_{0}:=h_{0}\left(z_{0}\right)$. Since $y_{0}$ is a regular value of $h \circ \bar{\varepsilon}^{-1}$ and $\bar{\varepsilon}(K)=\left(h \circ \bar{\varepsilon}^{-1}\right)^{-1}\left(y_{0}\right)$, it follows that the cohomology class $\left(h \circ \bar{\varepsilon}^{-1}\right)^{*}(v)$ is Poincaré dual to the homology class $[\bar{\varepsilon}(K)]_{Y}=\bar{\varepsilon}_{*}\left([K]_{L}\right)$ (cf. [27, Proposition 2.15]). Consequently, $r^{*}(v)$ is Poincaré dual to $\bar{\varepsilon}_{*}\left([K]_{L}\right)$, the maps $h \circ \bar{\varepsilon}^{-1}$ and $r$ being homotopic. Thus, $r^{*}(v) \cap[Y]=\bar{\varepsilon}_{*}\left([K]_{L}\right)$ and hence for every cohomology class $w$ in $H^{1}(Y ; \mathbb{Z} / 2)$,

$$
\left\langle w, \bar{\varepsilon}_{*}\left([K]_{L}\right)\right\rangle=\left\langle w, r^{*}(v) \cap[Y]\right\rangle=\left\langle w \cup r^{*}(v),[Y]\right\rangle .
$$

Since $r$ is a regular map and $r(Y) \subseteq \Sigma_{0}$, Example 2.3 implies that $r^{*}(v)$ is in $\operatorname{Alg}^{1}(Y)$. Hence, according to Theorem 2.4,

$$
H_{\mathrm{alg}}^{k}(Y ; \mathbb{Z} / 2) \subseteq\left\{w \in H^{k}(Y ; \mathbb{Z} / 2) \mid\left\langle w, \bar{\varepsilon}_{*}\left([K]_{L}\right)\right\rangle=0\right\},
$$

as required.

The ability to verify the bordism hypothesis in Lemma 2.5 is essential for applications. This often requires the following deep result from differential topology.

Theorem 2.6 (cf. [29, (17.3)]) Let $f: K \rightarrow P$ be a smooth map between compact smooth manifolds. The bordism class of $f$ in the group $\mathfrak{N}_{*}(P)$ is 0 if and only if for every nonnegative integer $q$ and every cohomology class $u$ in $H^{q}(P ; \mathbb{Z} / 2)$,

$$
\left\langle w_{i_{1}}(K) \cup \cdots \cup w_{i_{p}}(K) \cup f^{*}(u),[K]\right\rangle=0
$$

for all nonnegative integers $i_{1}, \ldots, i_{p}$ with $i_{1}+\cdots+i_{p}=k-q$, where $k=\operatorname{dim} K$.

Henceforth, the following notion will play a crucial role.

Definition 2.7 Given a compact smooth manifold $M$ and a subring $A$ of $H^{*}(M ; \mathbb{Z} / 2)$, a smooth submanifold $K$ of $M$ is said to be adapted to $A$ if for every nonnegative integer $q$ and every cohomology class $u$ in $A^{q}$,

$$
\left\langle w_{i_{1}}(K) \cup \cdots \cup w_{i_{p}}(K) \cup \kappa^{*}(u),[K]\right\rangle=0
$$

for all nonnegative integers $i_{1}, \ldots, i_{p}$ with $i_{1}+\ldots+i_{p}=k-q$, where $k=\operatorname{dim} K$ and $\kappa: K \hookrightarrow M$ is the inclusion map.

For any smooth manifold $N$, let $\tau_{N}$ denote its tangent bundle. 
Lemma 2.8 Let $M$ be a compact smooth manifold and let $K$ be a connected smooth submanifold of $M$ of positive dimension $k$, with $2 k+1 \leq \operatorname{dim} M$. If $K$ is adapted to a subring $A$ of $H^{*}(M ; \mathbb{Z} / 2)$ containing the Stiefel-Whitney classes $w_{i}(M)$ for $0 \leq i \leq k$, then the normal bundle of $K$ in $M$ splits off a trivial vector bundle of rank 2 .

Proof If $2 k+2 \leq \operatorname{dim} M$, then the assertion is true without any additional assumptions on $K$.

Suppose now that $2 k+1=\operatorname{dim} M$. It suffices to prove that the normal bundle $v$ of $K$ in $M$ has two continuous sections that are linearly independent at each point of $K$. Since rank $v=k+1$ and $\operatorname{dim} K=k$, the only obstruction to the existence of such sections is an element $W_{k}(v)$ in the cohomology group $H^{k}(X ; \Gamma)$, where $\Gamma$ is a local system of coefficients with fiber $\mathbb{Z}$ or $\mathbb{Z} / 2$ (cf. [52, p. 140] and [60, pp. 190, 191]).

If $k$ is even, then $\Gamma$ is isomorphic to the constant local system $\mathbb{Z} / 2$, and $W_{k}(v)$ can be identified with $w_{k}(v)$ (cf. [52, p. 143]).

If $k$ is odd, then the local system $\Gamma$ has fiber $\mathbb{Z}$. The group $H^{k}(K ; \Gamma)$ is isomorphic either to $\mathbb{Z}$ or $\mathbb{Z} / 2$. Indeed, the Poincaré duality gives an isomorphism between $H^{k}(K ; \Gamma)$ and the 0th homology group of $K$ with a suitable local system of coefficients with fiber $\mathbb{Z}$. The 0th homology group of $K$ with an arbitrary local system of coefficients with fiber $\mathbb{Z}$ is isomorphic either to $\mathbb{Z}$ or $\mathbb{Z} / 2$. If the group $H^{k}(K ; \Gamma)$ is infinite cyclic, then $W_{k}(v)=0$ since $W_{k}(v)$ is an element of order 2 (cf. [60, Theorem $38.11])$. If $H^{k}(K ; \Gamma)$ is isomorphic to $\mathbb{Z} / 2$, then the reduction modulo 2 homomorphism $\rho: H^{k}(K ; \Gamma) \rightarrow H^{k}(K ; \mathbb{Z} / 2)$ is an isomorphism. According to [52, Theorem 12.1], $\rho\left(W_{k}(v)\right)=w_{k}(v)$.

In conclusion, $W_{k}(\nu)=0$ if $w_{k}(\nu)=0$. It remains to prove the equality $w_{k}(\nu)=0$. If $\kappa: K \hookrightarrow M$ is the inclusion map, then the vector bundles $\tau_{K} \oplus \nu$ and $\kappa^{*}\left(\tau_{M}\right)$ are isomorphic, and hence, one gets $w(K) \cup w(v)=\kappa^{*}(w(M))$ for the total Stiefel-Whitney classes. The last equality implies that $w_{k}(\nu)$ belongs to the subring of $H^{*}(K ; \mathbb{Z} / 2)$ generated by $w_{i}(K)$ and $\kappa^{*}\left(w_{i}(M)\right)$ for $0 \leq i \leq k$. Consequently, $\left\langle w_{k}(v),[K]\right\rangle=0$ since $K$ is adapted to $A$ and $w_{i}(M)$ is in $A^{i}$ for $0 \leq i \leq k$. Thus, $w_{k}(v)=0$, the manifold $K$ being connected.

The next lemma is included for the sake of completeness. If $M$ is a compact smooth manifold and $N$ is a smooth submanifold of $M$ of codimension $k$, let $[N]^{M}$ denote the cohomology class in $H^{k}(M ; \mathbb{Z} / 2)$ that is Poincaré dual to the homology class $[N]_{M}$ represented by $N$. That is, $[N]^{M} \cap[M]=[N]_{M}$, where $\cap$ denotes the cap product.

Lemma 2.9 Let $M$ be a compact smooth manifold of dimension $m$. Let $K_{1}, \ldots, K_{p}$ be pairwise disjoint connected smooth submanifolds of $M$ of dimension $k$, where $1 \leq k \leq m-1$. Let $N$ be a smooth submanifold of $M$ of codimension $k$. If

$$
\left\langle[N]^{M},\left[K_{l}\right]_{M}\right\rangle=0 \text { for } 1 \leq l \leq p
$$

then there exists a smooth submanifold $N^{\prime}$ of $M$ of codimension $k$ such that $\left[N^{\prime}\right]^{M}=$ $[N]^{M}$ and $K_{l} \cap N^{\prime}=\emptyset$ for $1 \leq l \leq p$.

Proof Arguing by induction on the number of submanifolds $K_{l}$ suppose that $j$ is an integer satisfying $0 \leq j \leq p-1$, and $N_{j}$ is a smooth submanifold of $M$ with 
$\left[N_{j}\right]^{M}=[N]^{M}$ and $K_{l} \cap N_{j}=\varnothing$ for $1 \leq l \leq j$ (the last condition is vacuous if $j=0$ ). It suffices to prove the existence of a smooth submanifold $N_{j+1}$ of $M$ such that $\left[N_{j+1}\right]^{M}=[N]^{M}$ and $K_{l} \cap N_{j+1}=\emptyset$ for $1 \leq l \leq j+1$. The submanifold $N_{j}$ can be assumed to be transverse to $K_{l}$ for $1 \leq l \leq p$. Since

$$
\begin{aligned}
\left\langle\left[N_{j}\right]^{M} \cup\left[K_{j+1}\right]^{M},[M]\right\rangle & =\left\langle\left[N_{j}\right]^{M},\left[K_{j+1}\right]^{M} \cap[M]\right\rangle \\
& =\left\langle\left[N_{j}\right]^{M},\left[K_{j+1}\right]_{M}\right\rangle=0,
\end{aligned}
$$

the modulo 2 intersection number of $K_{j+1}$ and $N_{j}$ in $M$ is 0 , and hence, the set $K_{j+1} \cap N_{j}$ is either empty or consists of $2 r$ points for some positive integer $r$. In the former case, it suffices to set $N_{j+1}:=N_{j}$. In the latter case, let $x$ and $y$ be two points in $K_{j+1} \cap N_{j}$ that can be joined by a smooth $\operatorname{arc} C$ in $K_{j+1}$ satisfying $C \cap N_{j}=\{x, y\}$. The restriction to $C$ of the normal bundle of $K_{j+1}$ in $M$ is trivial, and hence making use of a thin $(m-k)$-dimensional tube along $C$, one can construct a smooth submanifold $N_{j}(x, y)$ of $M$ with $\left[N_{j}(x, y)\right]^{M}=\left[N_{j}\right]^{M}, K_{l} \cap N_{j}(x, y)=\emptyset$ for $1 \leq l \leq j$, and $K_{j+1} \cap N_{j}(x, y)=\left(K_{j+1} \cap N_{j}\right) \backslash\{x, y\}$. By repeating this procedure $r$ times, one obtains a smooth submanifold $N_{j+1}$ of $M$ having the required properties.

For any subring $A$ of $H^{*}(M ; \mathbb{Z} / 2)$, let

$$
A_{k}:=\left\{\alpha \in H_{k}(M ; \mathbb{Z} / 2) \mid\langle u, \alpha\rangle=0 \text { for all } u \in A^{k}\right\}
$$

Theorem 2.10 Let $M$ be a compact connected smooth manifold and let $r$ be a positive integer with $2 r+1 \leq \operatorname{dim} M$. Let $A$ be an $r$-admissible subring of $H^{*}(M ; \mathbb{Z} / 2)$ and let $\Delta$ be the set of all integers $k$ such that $1 \leq k \leq r$ and the group $A_{k}$ is generated by homology classes of the form $[K]_{M}$, where $K$ is a $k$-dimensional connected smooth submanifold of $M$ adapted to A. If $w_{i}(M)$ is in $A^{i}$ for $0 \leq i \leq r$, then there exists an algebraic model $(X, \varphi)$ of $M$ satisfying

$$
\varphi^{*}(A) \subseteq H_{\mathrm{alg}}^{*}(X ; \mathbb{Z} / 2) \text { and } \varphi^{*}\left(A^{k}\right)=H_{\mathrm{alg}}^{k}(X ; \mathbb{Z} / 2) \text { for } k \text { in }\{0\} \cup \Delta \text {. }
$$

Proof The subring $A$ can be written as $A=B[T]$, where $B$ is a full subring and $T$ is a regular subset of $H^{*}(M ; \mathbb{Z} / 2)$, with $T$ disjoint from $H^{c}(M ; \mathbb{Z} / 2)$ for $0 \leq c \leq r-1$. By definition,

$$
B=h^{*}\left(H^{*}(W ; \mathbb{Z} / 2)\right)
$$

where $h: M \rightarrow W$ is a smooth map into a compact smooth manifold $W$ with $A(W)=$ $H^{*}(W ; \mathbb{Z} / 2)$. In view of the last equality, the whole ring $H^{*}(W ; \mathbb{Z} / 2)$ is algebraically realizable (cf. Sect. 1), and hence, it can be assumed that $W$ is a nonsingular real algebraic variety satisfying

$$
H_{\mathrm{alg}}^{*}(W ; \mathbb{Z} / 2)=H^{*}(W ; \mathbb{Z} / 2)
$$

Let $m:=\operatorname{dim} M$. If $d$ is a sufficiently large integer and $G:=\mathbb{G}_{d, m}(\mathbb{R})$, then there exists a smooth classifying map $g: M \rightarrow G$ for the tangent bundle $\tau_{M}$ of $M$, that is, 
$\tau_{M}$ is isomorphic to the pullback $g^{*} \gamma$ of the universal vector bundle $\gamma$ on $G$. Hence, the subring $g^{*}\left(H^{*}(H ; \mathbb{Z} / 2)\right)$ of $H^{*}(M ; \mathbb{Z} / 2)$ is generated by $w_{i}(M)$ for $i \geq 0$. The smooth map $(g, h): M \rightarrow G \times W$ plays a crucial role. Set

$$
D:=(g, h)^{*}\left(H^{*}(G \times W ; \mathbb{Z} / 2)\right) \text { and } C:=D[T] .
$$

Since $w_{i}(M)$ is in $A^{i}$ for $0 \leq i \leq r$, by (1) and Künneth's theorem, the subring $C$ of $H^{*}(M ; \mathbb{Z} / 2)$ satisfies

$$
A \subseteq C \text { and } A^{i}=C^{i}, A_{i}=C_{i} \text { for } 0 \leq i \leq r
$$

By (2) and Proposition 2.2,

$$
\mathfrak{N}_{*}^{\text {alg }}(W)=\mathfrak{N}_{*}(W) \text { and } \mathfrak{N}_{*}^{\text {alg }}(G \times W)=\mathfrak{N}_{*}(G \times W) .
$$

In view of (4), if $p$ is a sufficiently large integer, then for each integer $k$ in $\Delta$, there exist $k$-dimensional connected smooth submanifolds $K_{k 1}, \ldots, K_{k p}$ of $M$ such that

$$
\begin{aligned}
& \text { each } K_{k l} \text { is adapted to } C \\
& {\left[K_{k 1}\right]_{M}, \ldots,\left[K_{k p}\right]_{M} \text { generate } C_{k}=A_{k} .}
\end{aligned}
$$

By (6) and Lemma 2.8,

the normal bundle of each $K_{k l}$ in $M$

$$
\text { splits off a trivial vector bundle of rank } 2 \text {. }
$$

If $\kappa_{k l}: K_{k l} \hookrightarrow M$ is the inclusion map, the restriction map $\left.(g, h)\right|_{K_{k l}}: K_{k l} \rightarrow$ $G \times W$ can be written as $\left.(g, h)\right|_{K_{k l}}=(g, h) \circ \kappa_{k l}$, and hence

$$
\begin{aligned}
\left(\left.(g, h)\right|_{K_{k l}}\right)^{*}\left(H^{*}(G \times W ; \mathbb{Z} / 2)\right)= \\
\kappa_{k l}^{*}\left((g, h)^{*}\left(H^{*}(G \times W ; \mathbb{Z} / 2)\right)\right) \subseteq \kappa_{k l}^{*}(C),
\end{aligned}
$$

where the inclusion follows from (3). Consequently, by (6) and Theorem 2.6,

$$
\text { the bordism class of }\left.(g, h)\right|_{K_{k l}}: K_{k l} \rightarrow G \times W \text { in } \mathfrak{N}_{*}(G \times W) \text { is } 0 \text {. }
$$

Let $N_{1}, \ldots, N_{q}$ be smooth submanifolds of $M$ such that

$$
T=\left\{\left[N_{1}\right]^{M}, \ldots,\left[N_{q}\right]^{M}\right\} \text { and } \operatorname{codim}_{M} N_{j} \geq r \text { for } 1 \leq j \leq q,
$$

and let

$$
N:=N_{1} \cup \cdots \cup N_{q} .
$$


The collection of smooth submanifolds of $M$ consisting of all $K_{k l}$ and all $N_{j}$ can be assumed to be in general position. In particular, the $K_{k l}$ are pairwise disjoint since $2 r+1 \leq m$. Similarly, each $K_{k l}$ with $1 \leq k \leq r-1$ is disjoint from $N$ since $\operatorname{codim}_{M} N_{j} \geq r$ for $1 \leq j \leq q$. Moreover, according to Lemma 2.9, the $N_{j}$ can be chosen in such a way that $K_{k l} \cap N=\emptyset$ for $k \in \Delta$ and $1 \leq l \leq p$.

One can assume that $M$ is smoothly embedded in $\mathbb{R}^{n}$ for some $n \geq 2 m+1$. Since (5) holds, according to [13, Theorem 4, Remark 8], it can be assumed that

$M$ is a nonsingular algebraic subset of $\mathbb{R}^{n}$,

$N_{1}, \ldots, N_{q}$ are nonsingular algebraic subsets of $M$,

$(g, h): M \rightarrow G \times W$ is a regular map.

Let $U_{k l}$ be a tubular neighborhood of $K_{k l}$ in $M$. The $U_{k l}$ can be chosen to be pairwise disjoint and disjoint from $N$. In view of (8), one can find a smooth embedding $\eta_{k l}: K_{k l} \times \mathbb{D}^{1} \rightarrow U_{k l}$ such that if $L_{k l}:=\eta_{k l}\left(K_{k l} \times \mathbb{S}^{1}\right)$ and $\theta_{k l}: K_{k l} \times \mathbb{S}^{1} \rightarrow L_{k l}$ is the smooth diffeomorphism determined by $\eta_{k l}$, then $K_{k l}=\theta_{k l}\left(K_{k l} \times\left\{z_{0}\right\}\right)$ for some point $z_{0}$ in $\mathbb{S}^{1}$. The smooth map $\left(\left.(g, h)\right|_{L_{k l}}\right) \circ \theta_{k l}: K_{k l} \times \mathbb{S}^{1} \rightarrow G \times W$ is a restriction of the smooth map $(g, h) \circ \eta_{k l}: K_{k l} \times \mathbb{D}^{1} \rightarrow G \times W$. Hence, by (9) and Lemma 2.5 (with $K=K_{k l}, L=L_{k l}$ and $f=\left.(g, h)\right|_{L_{k l}}$ ), there exist a smooth embedding $\varepsilon_{k l}: L_{k l} \rightarrow \mathbb{R}^{n}$, a nonsingular algebraic subset $Y_{k l}$ of $\mathbb{R}^{n}$, and a regular map $\left(g_{k l}, h_{k l}\right): Y_{k l} \rightarrow G \times W$ such that $Y_{k l}=\varepsilon_{k l}\left(L_{k l}\right), \varepsilon_{k l}$ is close to the inclusion map $L_{k l} \hookrightarrow \mathbb{R}^{n}$ in the space $\mathcal{C}^{\infty}\left(L_{k l}, \mathbb{R}^{n}\right),\left(g_{k l}, h_{k l}\right) \circ \bar{\varepsilon}_{k l}$ is close to $\left.(g, h)\right|_{L_{k l}}$ in $\mathcal{C}^{\infty}\left(L_{k l}, G \times W\right)$, and

$$
H_{\mathrm{alg}}^{k}\left(Y_{k l} ; \mathbb{Z} / 2\right) \subseteq\left\{w \in H^{k}\left(Y_{k l} ; \mathbb{Z} / 2\right) \mid\left\langle w, \bar{\varepsilon}_{k l *}\left(\left[K_{k l}\right]_{L_{k l}}\right)\right\rangle=0\right\},
$$

where $\bar{\varepsilon}_{k l}: L_{k l} \rightarrow Y_{k l}$ is the smooth diffeomorphism determined by $\varepsilon_{k l}$. If each $\left(g_{k l}, h_{k l}\right) \circ \bar{\varepsilon}_{k l}$ is sufficiently close to $\left.(g, h)\right|_{L_{k l}}$, then one can find a smooth map $\left(g^{\prime}, h^{\prime}\right): M \rightarrow G \times W$ that is homotopic to $(g, h): M \rightarrow G \times W$ and satisfies

$$
\begin{aligned}
\left.\left(g^{\prime}, h^{\prime}\right)\right|_{N}= & \left.(g, h)\right|_{N} \text { and } \\
& \left.\left(g^{\prime}, h^{\prime}\right)\right|_{L_{k l}}=\left(g_{k l}, h_{k l}\right) \circ \bar{\varepsilon}_{k l} \text { for } \in \Delta \text { and } 1 \leq l \leq p .
\end{aligned}
$$

If each $\varepsilon_{k l}$ is sufficiently close to the inclusion map $L_{k l} \hookrightarrow \mathbb{R}^{n}$, then

the $Y_{k l}$ are pairwise disjoint and disjoint from $N$,

and hence, there exists a smooth embedding $\varepsilon: M \rightarrow \mathbb{R}^{n}$ for which $\left.\varepsilon\right|_{L_{k l}}=\varepsilon_{k l}$ and $\left.\varepsilon\right|_{N}$ is the inclusion map $N \hookrightarrow \mathbb{R}^{n}$. Let $\bar{M}:=\varepsilon(M)$ and let $\bar{\varepsilon}: M \rightarrow \bar{M}$ be the smooth diffeomorphism determined by $\varepsilon$. The smooth map $(\bar{g}, \bar{h}):=\left(g^{\prime}, h^{\prime}\right) \circ \bar{\varepsilon}^{-1}: \bar{M} \rightarrow G \times W$ satisfies $\left.(\bar{g}, \bar{h})\right|_{Y_{k l}}=\left(g_{k l}, h_{k l}\right)$ and $\left.(\bar{g}, \bar{h})\right|_{N}=\left.\left(g^{\prime}, h^{\prime}\right)\right|_{N}$. Moreover, the algebraic subset

$$
Z:=N \cup \bigcup_{k, l} Y_{k l}
$$


of $\mathbb{R}^{n}$ is contained in $\bar{M}$, and by (12), (13), (15) and (16),

$$
\left.(\bar{g}, \bar{h})\right|_{Z}: Z \rightarrow G \times W \text { is a regular map. }
$$

Since $g: M \rightarrow G$ is a classifying map for $\tau_{M}$ and $g^{\prime}: M \rightarrow G$ is homotopic to $g$, it follows that $\bar{g}=g^{\prime} \circ \bar{\varepsilon}^{-1}: \bar{M} \rightarrow G$ is a classifying map for $\tau_{\bar{M}}$. Consequently, the regular map $\left.\bar{g}\right|_{Z}: Z \rightarrow G$ is a classifying map for $\left.\tau_{\bar{M}}\right|_{Z}$ and hence

$$
\left.\tau_{\bar{M}}\right|_{Z} \text { admits an algebraic structure }
$$

(cf. [16, Theorem 12.1.7]). In view of (5), (17) and (18), Theorem 2.1 can be applied to $\bar{h}: \bar{M} \rightarrow W$ and $Z \subseteq \bar{M}$. Therefore, there exist a smooth embedding $e: \bar{M} \rightarrow \mathbb{R}^{n}$, a nonsingular algebraic subset $X$ of $\mathbb{R}^{n}$ and a regular map $\lambda: X \rightarrow W$ such that $X=$ $e(\bar{M}), Z \subseteq X, e(x)=x$ for all $x$ in $Z$, and $\lambda \circ \bar{e}$ is homotopic to $\bar{h}$, where $\bar{e}: \bar{M} \rightarrow X$ is the smooth diffeomorphism determined by $e$. The map $\varphi:=\bar{\varepsilon}^{-1} \circ \bar{e}^{-1}: X \rightarrow M$ is a smooth diffeomorphism, and hence, $(X, \varphi)$ is an algebraic model of $M$.

By construction, $\lambda$ is homotopic to $\bar{h} \circ \bar{e}^{-1}=h^{\prime} \circ \varphi$ and $h^{\prime}$ is homotopic to $h$. Consequently, $\lambda$ is homotopic to $h \circ \varphi$, and hence,

$$
\lambda^{*}\left(H^{*}(W ; \mathbb{Z} / 2)\right)=\varphi^{*}\left(h^{*}\left(H^{*}(W ; \mathbb{Z} / 2)\right)\right)=\varphi^{*}(B),
$$

where the last equality follows from (1). This implies that

$$
\varphi^{*}(B) \subseteq H_{\mathrm{alg}}^{*}(X ; \mathbb{Z} / 2)
$$

since (2) holds and $\lambda: X \rightarrow W$ is a regular map. The diffeomorphism $\varphi: X \rightarrow M$ satisfies $\varphi(x)=x$ for all $x$ in $N$, which gives $\varphi^{*}\left(\left[N_{j}\right]^{M}\right)=\left[N_{j}\right]^{X}$ for $1 \leq j \leq q$. Thus, $\varphi^{*}\left(\left[N_{j}\right]^{M}\right)$ belongs to $H_{\mathrm{alg}}^{*}(X ; \mathbb{Z} / 2)$, each $N_{j}$ being an algebraic subset of $X$. By (10),

$$
\varphi^{*}(T) \subseteq H_{\mathrm{alg}}^{*}(X ; \mathbb{Z} / 2)
$$

The last two inclusions imply that

$$
\varphi^{*}(A) \subseteq H_{\mathrm{alg}}^{*}(X ; \mathbb{Z} / 2)
$$

Since $M$ is connected, one has $A^{0}=H^{0}(M ; \mathbb{Z} / 2)$ and

$$
\varphi^{*}\left(A^{0}\right)=H^{0}(X ; \mathbb{Z} / 2)=H_{\mathrm{alg}}^{0}(X ; \mathbb{Z} / 2)
$$

It remains to prove that if $u$ is a cohomology class in $H^{k}(M ; \mathbb{Z} / 2) \backslash A^{k}$ for some $k \in \Delta$, then $\varphi^{*}(u)$ is not in $H_{\text {alg }}^{k}(X ; \mathbb{Z} / 2)$. Let $\delta_{k l}: Y_{k l} \hookrightarrow X$ be the inclusion map. The composite map

$$
\varphi \circ \delta_{k l} \circ \bar{\varepsilon}_{k l}=\bar{\varepsilon}^{-1} \circ \bar{e}^{-1} \circ \delta_{k l} \circ \bar{\varepsilon}_{k l}: L_{k l} \rightarrow M
$$


is the inclusion map $L_{k l} \hookrightarrow M$, and hence,

$$
\left\langle\delta_{k l}^{*}\left(\varphi^{*}(u)\right), \bar{\varepsilon}_{k l *}\left(\left[K_{k l}\right]_{L_{k l}}\right)\right\rangle=\left\langle u,\left(\varphi \circ \delta_{k l} \circ \bar{\varepsilon}_{k l}\right)_{*}\left(\left[K_{k l}\right]_{L_{k l}}\right)\right\rangle=\left\langle u,\left[K_{k l}\right]_{M}\right\rangle .
$$

Since $u$ is not in $A^{k}$, condition (7) implies the existence of $l$ with $\left\langle u,\left[K_{k l}\right]_{M}\right\rangle \neq 0$. For this $l$, according to (14), $\delta_{k l}^{*}\left(\varphi^{*}(u)\right)$ is not in $H_{\mathrm{alg}}^{k}\left(Y_{k l} ; \mathbb{Z} / 2\right)$. Consequently, $\varphi^{*}(u)$ is not in $H_{\mathrm{alg}}^{k}(X ; \mathbb{Z} / 2)$, the map $\delta_{k l}$ being regular. The proof is complete.

Recall that a compact smooth manifold is said to be a boundary if it is diffeomorphic to the boundary of a compact smooth manifold with boundary.

Let $M$ be a compact connected smooth manifold and let $K$ be a smooth submanifold of $M$. If $K$ is adapted to a subring of $H^{*}(M ; \mathbb{Z} / 2)$, then the Stiefel-Whitney numbers of $K$ are all 0 , and hence, $K$ is a boundary [62]. Conversely, if $K$ is a boundary, then its Stiefel-Whitney numbers are all 0 , and hence, $K$ is adapted to the subring $H^{0}(M ; \mathbb{Z} / 2)$ of $H^{*}(M ; \mathbb{Z} / 2)$. The last observation can be generalized. This is done in the following two lemmas, in which notation $M$ and $K$ is preserved, and $k:=\operatorname{dim} K$ is assumed to be positive. Moreover, $A$ denotes a subring of $H^{*}(M ; \mathbb{Z} / 2)$.

Lemma 2.11 Assume that the submanifold $K$ is a boundary and the cohomology class $[K]_{M}$ belongs to $A_{k}$. Then, $K$ is adapted to $A$ if one of the following two conditions is satisfied:

(c) $1 \leq k \leq 2$.

(c) $k \geq 3, \mathrm{Sq}^{1}\left(A^{k-1}\right) \subseteq A^{k}$, and $A^{i}=0$ for $1 \leq i \leq k-2$.

Proof By Wu's theorem [52, Theorem 11.14], the first Wu class of $K$ is equal to $w_{1}(K)$. In particular,

$$
\operatorname{Sq}^{1}(a)=w_{1}(K) \cup a \text { for all } a \text { in } H^{k-1}(K ; \mathbb{Z} / 2) .
$$

Let $\kappa: K \hookrightarrow M$ be the inclusion map. Since $[K]_{M}$ is in $A_{k}$, for every cohomology class $z$ in $A^{k}$,

$$
\left\langle\kappa^{*}(z),[K]\right\rangle=\left\langle z, \kappa_{*}([K])\right\rangle=\left\langle z,[K]_{M}\right\rangle=0 .
$$

According to (1), for every cohomology class $v$ in $A^{k-1}$,

$$
w_{1}(K) \cup \kappa^{*}(v)=\operatorname{Sq}^{1}\left(\kappa^{*}(v)\right)=\kappa^{*}\left(\operatorname{Sq}^{1}(v)\right) .
$$

Therefore, the inclusion $\operatorname{Sq}^{1}\left(A^{k-1}\right) \subseteq A^{k}$ (which is automatically satisfied if $1 \leq k \leq 2$ ) and (2) give

$$
\left\langle w_{1}(K) \cup \kappa^{*}(v),[K]\right\rangle=0 .
$$

Since $K$ is a boundary, the Stiefel-Whitney numbers of $K$ are all 0 . Consequently, in view of (2) and (3), the submanifold $K$ is adapted to $A$, provided that either $\left(\mathrm{c}_{1}\right)$ or $\left(c_{2}\right)$ is satisfied. 
Lemma 2.12 Assume that the submanifold $K$ is a boundary and the homology class $[K]_{M}$ belongs to $A_{k}$. Moreover, assume that $K$ is orientable. Then, $K$ is adapted to $A$ if one of the following two conditions is satisfied:

(c 1 ) $1 \leq k \leq 4$.

$\left(\mathrm{c}_{2}\right) k \geq 5, \mathrm{Sq}^{2}\left(A^{k-2}\right) \subseteq A^{k}, \mathrm{Sq}^{2}\left(\mathrm{Sq}^{1}\left(A^{k-3}\right)\right) \subseteq A^{k}$, and $A^{i}=0$ for $1 \leq i \leq k-4$.

Proof In view of Lemma 2.11, it can be assumed that $k \geq 3$. Let $v_{j}(K)$ denote the $j$ th Wu class of $K$. The orientability of $K$ implies that

$$
w_{1}(K)=0
$$

and hence by Wu's theorem [52, Theorem 11.14], $v_{1}(K)=0$ and $v_{2}(K)=w_{2}(K)$. In particular,

$$
\begin{aligned}
& \operatorname{Sq}^{1}(a)=v_{1}(K) \cup a=0 \text { for all } a \text { in } H^{k-1}(K ; \mathbb{Z} / 2) \\
& \operatorname{Sq}^{2}(b)=v_{2}(K) \cup b=w_{2}(K) \cup b \text { for all } b \text { in } H^{k-2}(K ; \mathbb{Z} / 2) .
\end{aligned}
$$

Let $\kappa: K \hookrightarrow M$ be the inclusion map. Since $[K]_{M}$ is in $A_{k}$, for every cohomology class $z$ in $A^{k}$,

$$
\left\langle\kappa^{*}(z),[K]\right\rangle=\left\langle z, \kappa_{*}([K])\right\rangle=\left\langle z,[K]_{M}\right\rangle=0 .
$$

According to (3), for every cohomology class $v$ in $H^{k-2}(M ; \mathbb{Z} / 2)$,

$$
w_{2}(K) \cup \kappa^{*}(v)=\operatorname{Sq}^{2}\left(\kappa^{*}(v)\right)=\kappa^{*}\left(\operatorname{Sq}^{2}(v)\right) .
$$

If $\operatorname{Sq}^{2}(v)$ is in $A^{k}$ (which is automatically satisfied if $3 \leq k \leq 4$ ), then (4) gives

$$
\left\langle w_{2}(K) \cup \kappa^{*}(v),[K]\right\rangle=0 .
$$

According to (2), for every cohomology class $u$ in $H^{k-3}(M ; \mathbb{Z} / 2)$,

$$
\mathrm{Sq}^{1}\left(w_{2}(K) \cup \kappa^{*}(u)\right)=0 .
$$

On the other hand,

$$
\mathrm{Sq}^{1}\left(w_{2}(K) \cup \kappa^{*}(u)\right)=\operatorname{Sq}^{1}\left(w_{2}(K)\right) \cup \kappa^{*}(u)+w_{2}(K) \cup \kappa^{*}\left(\operatorname{Sq}^{1}(u)\right) .
$$

Consequently,

$$
\mathrm{Sq}^{1}\left(w_{2}(K)\right) \cup \kappa^{*}(u)=w_{2}(K) \cup \kappa^{*}\left(\mathrm{Sq}^{1}(u)\right)
$$

By Wu's formula [52, p. 94], $\operatorname{Sq}^{1}\left(w_{2}(K)\right)=w_{1}(K) \cup w_{2}(K)+w_{3}(K)$, which in view of $(1)$ gives $\operatorname{Sq}^{1}\left(w_{2}(K)\right)=w_{3}(K)$. Thus,

$$
w_{3}(K) \cup \kappa^{*}(u)=\operatorname{Sq}^{1}\left(w_{2}(K)\right) \cup \kappa^{*}(u)=w_{2}(K) \cup \kappa^{*}\left(\operatorname{Sq}^{1}(u)\right) .
$$


If $\mathrm{Sq}^{2}\left(\mathrm{Sq}^{1}(u)\right)$ is in $A^{k}$ (which is automatically satisfied if $3 \leq k \leq 4$ ), then (5) gives

$$
\left\langle w_{3}(K) \cup \kappa^{*}(u),[K]\right\rangle=0 .
$$

Since $K$ is a boundary, the Stiefel-Whitney numbers of $K$ are all 0 . Consequently, in view of (1), (4), (5) and (6), the submanifold $K$ is adapted to $A$, provided that either $\left(c_{1}\right)$ or $\left(c_{2}\right)$ is satisfied.

The assumption in Lemmas 2.11 and 2.12 that $K$ be a boundary is not a serious limitation for applications, as demonstrated below.

The following is a simple modification of a deep result of Thom [62, Théorème II.26].

Lemma 2.13 (cf. [40, Lemma 4.7]) Let $M$ be a compact connected smooth manifold and let $k$ be a positive integer satisfying $2 k \leq \operatorname{dim} M$. Then, each homology class in $H_{k}(M ; \mathbb{Z} / 2)$ is of the form $[K]_{M}$, where $K$ is a $k$-dimensional connected smooth submanifold of $M$. Moreover, $K$ can be chosen in such a way that it is a boundary.

Under some additional assumptions, $K$ can be assumed to be orientable.

Lemma 2.14 Let $M$ be a compact connected smooth manifold and let $k$ be a positive integer satisfying $2 k+1 \leq \operatorname{dim} M$. Assume that the homology group $H_{k-1}(M ; \mathbb{Z})$ has no 2-torsion. Then, each homology class in $H_{k-1}(M ; \mathbb{Z} / 2)$ is of the form $[K]_{M}$, where $K$ is a $k$-dimensional connected orientable smooth submanifold of M. Moreover, $K$ can be chosen in such a way that it is a boundary.

Proof By the universal coefficient theorem, the reduction modulo 2 homomorphism $\rho: H_{k}(M, \mathbb{Z}) \rightarrow H_{k}(M, \mathbb{Z} / 2)$ is surjective. Hence, each homology class $\alpha$ in $H_{k}(M ; \mathbb{Z} / 2)$ is of the form $\alpha=\rho(\beta)$ for some homology class $\beta$ in $H_{k}(M ; \mathbb{Z})$. According to [29, Corollary 15.3], one can find a $k$-dimensional oriented compact smooth manifold $N$, a smooth map $f: N \rightarrow M$ and an integer $r$ such that $f_{*}\left(\mu_{N}\right)=(2 r+1) \beta$, where $\mu_{N}$ is the fundamental class of $N$ in $H_{k}(N, \mathbb{Z})$. Since $2 k+1 \leq m:=\operatorname{dim} M$, the map $f$ can be assumed to be a smooth embedding (cf. [30, Theorem 2.13]). Hence, $P:=f(N)$ is an orientable smooth submanifold of $M$ with $[P]_{M}=\alpha$. By joining the connected components of $P$ with $k$-dimensional tubes in $M$, one obtains an orientable connected smooth submanifold $L$ of $M$ satisfying $[L]_{M}=\alpha$. Let $U$ be an open subset of $M \backslash L$, diffeomorphic to $\mathbb{R}^{m}$. Let $L^{\prime}$ be a smooth submanifold of $U$, diffeomorphic to $L$. By joining $L$ and $L^{\prime}$ with a $k$-dimensional tube in $M$, one gets an orientable connected smooth submanifold $K$ of $M$ satisfying $[K]_{M}=\alpha$. By construction, $K$ is a boundary.

One more observation is required for the proofs of the main results.

Lemma 2.15 Let $M$ be a compact smooth manifold and let $A$ be an $r$-admissible subring of $H^{*}(M ; \mathbb{Z} / 2)$, where $r$ is a positive integer. Then, $\operatorname{Sq}^{i}\left(A^{j}\right) \subseteq A^{i+j}$ for all nonnegative integers $i$ and $j$ with $j \leq r-1$.

Proof It suffices to observe that for each full subring $B$ of $H^{*}(M ; \mathbb{Z} / 2)$, one has $\mathrm{Sq}^{i}\left(B^{j}\right) \subseteq B^{i+j}$ for all nonnegative integers $i$ and $j$. 
Proof of Theorem 1.1 It is already known that (a) implies (b). If $k$ is an integer satisfying $1 \leq k \leq r$, then according to Lemma 2.13, each homology class in $A_{k}$ is of the form $[K]_{M}$, where $K$ is a $k$-dimensional connected smooth submanifold of $M$ that is a boundary. By Lemmas 2.11 and $2.15, K$ is adapted to $A$. Hence, (b) implies (a) in view of Theorem 2.10.

Proof of Theorem 1.7 If $k$ is an integer satisfying $1 \leq k \leq r$, then according to Lemma 2.13, each homology class in $A_{k}$ is of the form $[K]_{M}$, where $K$ is a $k$-dimensional connected smooth submanifold of $M$ that is a boundary. Moreover, according to Lemma $2.14, K$ can be assumed to be an orientable manifold if the homology group $H_{k-1}(M ; \mathbb{Z})$ has no 2-torsion. By Lemmas 2.11 and $2.15, K$ is adapted to $A$ if $k$ is in $\{1, \ldots, r-2\} \cup\{2\}$. By Lemmas 2.12 and $2.15, K$ is adapted to $A$ if $r \geq 4$ and either $A^{r-3}=0$ or the homology group $H_{r-2}(M ; \mathbb{Z})$ has no 2 -torsion. The proof is complete in view of Theorem 2.10.

Open Access This article is distributed under the terms of the Creative Commons Attribution Noncommercial License which permits any noncommercial use, distribution, and reproduction in any medium, provided the original author(s) and source are credited.

\section{References}

1. Akbulut, S., King, H.: The topology of real algebraic sets with isolated singularities. Ann. Math. 113, 425-446 (1981)

2. Akbulut, S., King, H.: A relative Nash theorem. Trans. Am. Math. Soc. 267, 465-481 (1981)

3. Akbulut, S., King, H.: The topology of real algebraic sets. Enseign. Math. 29, 221-261 (1983)

4. Akbulut, S., King, H.: Submanifolds and homology of nonsingular real algebraic varieties. Am. J. Math. 107, 45-83 (1985)

5. Akbulut, S., King, H.: A resolution theorem for homology cycles of real algebraic varieties. Invent. Math. 79, 589-601 (1985)

6. Akbulut, S., King, H.: On approximating submanifolds by algebraic sets and a solution to the Nash conjecture. Invent. Math. 107, 87-98 (1992)

7. Akbulut, S., King, H.: Algebraicity of immersions in $\mathbb{R}^{n}$. Topology 31, 701-712 (1992)

8. Akbulut, S., King, H.: Topology of Real Algebraic Sets, Math. Sci. Res. Inst. Publ. 25, Springer (1992)

9. Akbulut, S., King, H.: Transcendental submanifolds of $\mathbb{R}^{n}$. Comment. Math. Helv. 68, 308-318 (1993)

10. Akbulut, S., King, H.: Transcendental submanifolds of $\mathbb{R} P^{n}$. Comment. Math. Helv. 80, 427-432 (2005)

11. Benedetti, R.: On a resolution theorem for homology classes of a real algebraic variety. Boll. Un. Mat. Ital. A(6) 4, 459-466 (1985)

12. Benedetti, R., Dedò, M.: Counterexamples to representing homology classes by real algebraic subvarieties up to homeomorphism. Compos. Math. 53, 143-151 (1984)

13. Benedetti, R., Tognoli, A.: Approximation theorems in real algebraic geometry. Boll. Un. Mat. Ital. Suppl. 2, 209-228 (1980)

14. Benedetti, R., Tognoli, A.: On real algebraic vector bundles. Bull. Sci. Math.(2) 104, 89-112 (1980)

15. Benedetti, R., Tognoli, A.: Remarks and counterexamples in the theory of real vector bundles and cycles. In: Géométrie algebrique réelle et formes quadratiques, Lecture Notes in Math. 959, Springer, 198-211 (1982)

16. Bochnak, J., Coste, M., Roy, M.-F.: Real Algebraic Geometry, Ergeb. Math. Grenzgeb. 36. Springer, Berlin (1998)

17. Bochnak, J., Kucharz, W.: Algebraic models of smooth manifolds. Invent. Math. 97, 585-611 (1989)

18. Bochnak, J., Kucharz, W.: $K$-theory of real algebraic surfaces and threefolds. Math. Proc. Camb. Philos. Soc. 106, 471-480 (1989)

19. Bochnak, J., Kucharz, W.: Nonisomorphic algebraic models of a smooth manifold. Math. Ann. 290, 1-2 (1991) 
20. Bochnak, J., Kucharz, W.: Algebraic cycles and approximation theorems in real algebraic geometry. Trans. Am. Math. Soc. 337, 463-472 (1993)

21. Bochnak, J., Kucharz, W.: Real algebraic hypersurfaces in complex projective varieties. Math. Ann. 301, 381-397 (1995)

22. Bochnak, J., Kucharz, W.: On homology classes represented by real algebraic varieties. In: Singularities Symposium—Łojasiewicz 70, Banach Center Publ. 44, Inst. Math., Polish Acad. Sci. 21-35 (1998)

23. Bochnak, J., Kucharz, W.: A topological proof of the Grothendieck formula in real algebraic geometry. Enseign. Math. 48, 237-258 (2002)

24. Bochnak, J., Kucharz, W.: On approximation of smooth manifolds by nonsingular real algebraic subvarieties. Ann. Sci. École Norm. Sup. (4) 36, 685-690 (2003)

25. Bochnak, J., Kucharz, W.: Real algebraic morphisms represent few homotopy classes. Math. Ann. 337, 909-921 (2007)

26. Bochnak, J., Kucharz, W., Shiota, M.: On algebraicity of global real analytic sets and functions. Invent. Math. 70, 115-156 (1982/1983)

27. Borel, A., Haefliger, A.: La classe d'homologie fondamentale d'un espace analytique. Bull. Soc. Math. France 89, 461-513 (1961)

28. Bröcker, L.: Reelle Divisoren. Arch. Math. (Basel) 35, 140-143 (1980)

29. Conner, P.E.: Differentiable Periodic Maps, 2nd edn, Lecture Notes in Math. 738. Springer (1979)

30. Hirsch, M.: Differential Topology, Graduate Texts in Math. vol. 33. Springer, New York, Heidelberg, Berlin (1976)

31. Huisman, J.: Real abelian varieties with complex multiplication, Ph.D. Thesis, Vrije Universiteit Amsterdam (1992)

32. Husemoller, D.: Fibre Bundles. Springer, Berlin, New York (1975)

33. Ischebeck, F., Schülting, H.-W.: Rational and homology equivalence for real cycles. Invent. Math. 94, 307-316 (1988)

34. Ivanov, N.: Approximation of smooth manifolds by real algebraic sets. Russ. Math. Surv. 37, 1-59 (1982)

35. King, H.: Approximating submanifolds of real projective space by varieties. Topology 15, 81-84 (1976)

36. Krasnov, V.A.: On the equivariant Grothendieck cohomology of a real algebraic variety and its application. Izv. Ross. Akad. Nauk Ser. Mat. 58, 36-52 (1994) (Russian); English transl. Russ. Acad. Sci. Izv. Math. 44, 461-477 (1995)

37. Kucharz, W.: On homology of real algebraic sets. Invent. Math. 82, 19-26 (1985)

38. Kucharz, W.: Algebraic equivalence and homology classes of real algebraic cycles. Math. Nachr. 180, 135-140 (1996)

39. Kucharz, W.: Algebraic morphisms into rational real algebraic surfaces. J. Algebraic Geom. 8, 569-579 (1999)

40. Kucharz, W.: Algebraic cycles and algebraic models of smooth manifolds. J. Algebraic Geom. 11, 101127 (2002)

41. Kucharz, W.: Nash cohomology of smooth manifolds. Ann. Polon. Math. 87, 193-205 (2005)

42. Kucharz, W.: Homology classes of real algebraic sets. Ann. Inst. Fourier (Grenoble) 58, 989$1022(2008)$

43. Kucharz, W.: Transcendental submanifolds of projective space. Comment. Math. Helv. 84, 127-133 (2009)

44. Kucharz, W.: Cycles on Nash algebraic models of smooth manifolds. Proc. Am. Math. Soc. 137, 1899_ 1906 (2009)

45. Kucharz, W.: Cycles on algebraic models of smooth manifolds. J. Eur. Math. Soc. (JEMS) 11, 393-405 (2009)

46. Kucharz, W.: Rational maps in real algebraic geometry. Adv. Geom. 9, 517-539 (2009)

47. Kucharz, W., Kurdyka, K.: Algebraicity of global real analytic hypersurfaces. Geom. Dedic. 119, 141149 (2006)

48. Kucharz, W., van Hamel, J.: Transcendental manifolds in real projective space and Stiefel-Whitney classes. Ann. Sc. Norm. Super. Pisa Cl. Sci. 5(8), 267-277 (2009)

49. Mangolte, F.: Cycles algébriques sur les surfaces $K 3$ réelles. Math. Z. 225, 559-576 (1995)

50. Mangolte, F.: Cycles algébriques et topologie des surfaces bielliptiques réelles. Comment. Math. Helv. 78, 385-393 (2003) 
51. Mangolte, F., van Hamel, J.: Algebraic cycles and topology of real Enriques surfaces. Compos. Math. 110, 215-237 (1998)

52. Milnor, J., Stasheff, J.: Characteristic Classes. Ann. Math. Stud. 76. Princeton University Press, Princeton, NJ (1974)

53. Nash, J.: Real algebraic manifolds. Ann. Math. 56, 405-421 (1952)

54. Risler, J.-J.: Sur l'homologie des surfaces algébriques réelles. In: Géométrie algébrique réelle et formes quadratiques, Lecture Notes in Math. 959, 381-385. Springer (1982)

55. Shiota, M.: Equivalence of differentiable functions, rational functions and polynomials. Ann. Inst. Fourier (Grenoble) 32, 167-204 (1982)

56. Shiota, M.: Real algebraic realization of characteristic classes. Publ. Res. Inst. Math. Sci. 18, 9951008 (1982)

57. Silhol, R.: A bound on the order of $H_{n-1}^{a}(X, \mathbb{Z} / 2)$ on a real algebraic variety. In: Géométrie algébrique réelle et formes quadratiques, Lecture Notes in Math. 959, 443-450. Springer (1982)

58. Silhol, R.: Real Algebraic Surfaces, Lecture Notes in Math. 1392. Springer (1989)

59. Spainer, E.: Algebraic Topology. Springer, New York (1966)

60. Steenrod, N.: The Topology of Fibre Bundles. Princeton University Press, Princeton, NJ (1951)

61. Teichner, P.: 6-dimensional manifolds without totally algebraic homology. Proc. Am. Math. Soc. 123, 2909-2914 (1995)

62. Thom, R.: Quelques propriétes globales de variétés différentiables. Comment. Math. Helv. 28, 17$86(1954)$

63. Tognoli, A.: Su una congettura di Nash. Ann. Sc. Norm. Sup. Pisa Sci. Fis. Mat. 3(27), 167-185 (1973)

64. Tognoli, A.: Algebraic approximation of manifolds and spaces. In: Séminaire Bourbaki, 32e année, 1979/1980, no. 548, Lecture Notes in Math. vol. 842, 73-94. Springer (1981)

65. van Hamel, J.: Real algebraic cycles on complex varieties. Math. Z. 225, 177-198 (1997)

66. van Hamel, J.: Algebraic Cycles and Topology of Real Algebraic Varieties, CWI Tract, vol. 129. Stichting Mathematisch Centrum, Amsterdam (2000) 